\title{
ARTIFICIAL CONDITIONS FOR THE LINEAR ELASTICITY EQUATIONS
}

\author{
VIRGINIE BONNAILLIE-NOËL, MARC DAMBRINE, FRÉDÉRIC HÉRAU, \\ AND GRÉGORY VIAL
}

\begin{abstract}
In this paper, we consider the equations of linear elasticity in an exterior domain. We exhibit artificial boundary conditions on a circle, which lead to a non-coercive second order boundary value problem. In the particular case of an axisymmetric geometry, explicit computations can be performed in Fourier series proving the well-posedness except for a countable set of parameters. A perturbation argument allows us to consider near-circular domains. We complete the analysis by some numerical simulations.
\end{abstract}

\section{INTRODUCTION}

We are considering a material whose core contains some bubbles of void. The presence of these inhomogenities weaken the material and the behavior of the device under loading is a major issue: will it crack? There are several difficulties in modeling the nucleation of a crack in a sound material. First, one has to propose a law able to predict that nucleation: one can use the Griffith criterion or an energy based criterion like the one proposed by Francfort and Marigo 11. On another hand we must determine the influence of geometric perturbations where a crack can initiate. To this end, we can perform a multiscale asymptotic analysis of the equations of elasticity for a linear isotropic material with Hooke's law $H$. Recall that for any symmetric matrix $e, H$ is defined by

$$
H e=\lambda \operatorname{tr}(e) \operatorname{Id}+2 \mu e,
$$

where $\mu$ and $\lambda$ are the Lamé constants of the material. In this work, we are more particularly interested in the numerical computation of corrective terms appearing in the evaluation of stress concentration due to the presence of geometrical defects.

Let $\Omega_{0}$ be a domain of $\mathbb{R}^{2}$ containing the origin 0 . We restrict ourselves to the bidimensional case in order to reduce the technicality of the paper. We consider a domain $\Omega_{\varepsilon}$ punctured with some inhomogeneities of size $\varepsilon$ near well separated points $x^{j}$ (see Figure 1): for $N$ defects it can be defined as

$$
\Omega_{\varepsilon}=\Omega_{0} \backslash \bigcup_{j=1}^{N} \overline{\omega_{\varepsilon}^{j}}, \quad \text { with } \quad \omega_{\varepsilon}^{j}=x^{j}+\varepsilon \omega^{j} .
$$

Received by the editor December 10, 2012 and, in revised form, September 4, 2013 and October 7, 2013.

2010 Mathematics Subject Classification. Primary 35J47, 35J57, 35P10, 35S15, 47A10, 47G30, $65 \mathrm{~N} 20$.

Key words and phrases. Linear elasticity equations, singular perturbation, artificial boundary conditions, Ventcel condition, Dirichlet-to-Neumann map, spectral theory. 
The case of a single perturbation was presented in [37. The case of two relatively close inclusions was studied in [8, 36].

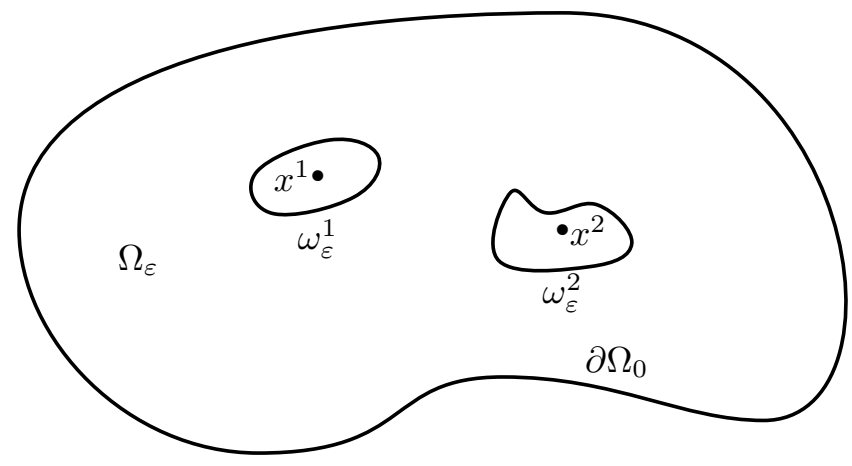

Figure 1. The perturbed domain when $N=2$.

Of course, the size of the unperturbed domain $\Omega_{0}$ does not depend on the parameter $\varepsilon$. We assume that the reference domains $\omega^{j}$ contain the origin 0 . Their dilated and translated copies $\omega_{\varepsilon}^{j}$ represent the defects. We denote by $\mathbf{H}_{\infty}^{j}$ the unbounded domains obtained by a blow-up around each perturbation:

$$
\mathbf{H}_{\infty}^{j}=\mathbb{R}^{2} \backslash \overline{\omega^{j}} .
$$

The problem we focus on is written on the perturbed domain as:

$$
\left\{\begin{aligned}
-\operatorname{div} \sigma\left(\mathbf{u}_{\varepsilon}\right)=-\mu \Delta \mathbf{u}_{\varepsilon}-(\lambda+\mu) \nabla \operatorname{div} \mathbf{u}_{\varepsilon} & =\mathbf{0} & & \text { in } \Omega_{\varepsilon}, \\
\mathbf{u}_{\varepsilon} & =\mathbf{u}^{d} & & \text { on } \Gamma_{d}, \\
\sigma\left(\mathbf{u}_{\varepsilon}\right) \cdot \mathbf{n} & =\mathbf{g} & & \text { on } \Gamma_{n},
\end{aligned}\right.
$$

where $\mathbf{u}_{\varepsilon}$ denotes the displacement and $\sigma\left(\mathbf{u}_{\varepsilon}\right)=H e\left(\mathbf{u}_{\varepsilon}\right)$ and $e\left(\mathbf{u}_{\varepsilon}\right)$ stand respectively for the stress tensor and the linearized strain tensor defined by

$\sigma(\mathbf{u})=\lambda \operatorname{tr}(e(\mathbf{u})) \operatorname{Id}+2 \mu e(\mathbf{u}), \quad e(\mathbf{u})=\frac{1}{2}\left[D \mathbf{u}+D \mathbf{u}^{\top}\right] \quad$ with $\quad(D \mathbf{u})_{i, j}=\partial_{i} u_{j}$.

$\Gamma_{d}$ and $\Gamma_{n}$ are the disjoint Dirichlet and Neumann boundaries of the domain respectively. $\Gamma_{n}$ includes the boundary of the perturbation and the loading $\mathbf{g}$ acts on the outer boundary; hence it is supposed to be zero in a neighborhood of the perturbation. This problem enters the general framework of local perturbations for elliptic problems, which have been deeply studied. Among others, let us mention the following works using potential theory [3, 5,38 , 38 and the reference monographs $[30,31$ for multiscale expansions. Following [10,36], the solution of (1.1) is given to first order by

$$
\mathbf{u}_{\varepsilon}(x)=\mathbf{u}_{0}(x)-\varepsilon \sum_{j=1}^{N}\left[\alpha_{1} \mathbf{v}_{1}^{j}\left(\frac{x-x^{j}}{\varepsilon}\right)+\alpha_{2} \mathbf{v}_{2}^{j}\left(\frac{x-x^{j}}{\varepsilon}\right)\right]+\mathcal{O}\left(\varepsilon^{2}\right),
$$

with $\mathbf{u}_{0}$ the solution on the unperturbed domain, $\boldsymbol{\alpha}_{1}=\sigma_{11}\left(\mathbf{u}_{0}\right)(0)$ and $\boldsymbol{\alpha}_{2}=$ $\sigma_{12}\left(\mathbf{u}_{0}\right)(0)$. When the distance between the $x^{j}$ cannot be assumed large with respect to $\varepsilon$, e.g. when $\left\|x^{i}-x^{j}\right\| \approx \varepsilon^{\alpha}$ for $\alpha \in(0,1)$, the order of the error term in (1.2) is reduced (see [10]). 
The profiles $\mathbf{v}_{1}^{j}$ and $\mathbf{v}_{2}^{j}$ are obtained as solutions of a homogeneous Navier equation posed on the unbounded domain $\mathbf{H}_{\infty}^{j}$ with Neumann conditions on the boundary of the normalized perturbation:

$$
\left\{\begin{array}{rlll}
-\mu \Delta \mathbf{v}_{\ell}-(\lambda+\mu) \nabla \operatorname{div} \mathbf{v}_{\ell} & =\mathbf{0} & & \text { in } \mathbf{H}_{\infty} \\
\sigma\left(\mathbf{v}_{\ell}\right) \cdot \mathbf{n} & =\mathbf{G}_{\ell} & & \text { on } \partial \omega \\
\mathbf{v}_{\ell} & \rightarrow \mathbf{0} & \text { at infinity }
\end{array}\right.
$$

with $\mathbf{G}_{1}=\left(\mathbf{n}_{1}, 0\right), \mathbf{G}_{2}=\left(0, \mathbf{n}_{1}\right)$ and $\mathbf{n}_{1}$ the first component of the outer normal to $\partial \mathbf{H}_{\infty}$ for $\omega=\omega_{j}$. Note that these profiles or correctors depend only on the shapes of the defects and not on their positions $x^{j}$, on the size $\varepsilon$ of the defects or on the loading $\mathbf{g}$.

In the spirit of XFEM methods, we can enrich the usual FEM space on a coarse mesh by these first order correctors $\mathbf{v}_{\ell}^{j}$. For a general shape $\omega^{j}$, there is no explicit solution to (1.3) and a numerical computation is needed. In this work, we propose and study a method to perform this computation.

Since problem (1.3) is posed on an infinite domain (an exterior domain in the present case), its numerical approximation is not straightforward. Among the techniques known to overcome this difficulty, let us mention the method of infinite elements, introduced in the seventies (see [7, 39]), which directly handles the problem with a standard Galerkin formulation. The other (numerous) methods reduce to a bounded domain for a classical finite element resolution. This is the case of absorbing conditions (mainly for wave propagation), [16, 18, 23, 25, 35. or integral representation, 27. In both cases, the domain is reduced to a ball, on the boundary of which a new condition - which is non-local most of the time - is imposed to get an equivalent formulation.

The present work is in line with such techniques, but we restrict ourselves to differential conditions and therefore seek approximate boundary conditions on the artificial boundary. Here we choose the boundary to be the circle $\partial \mathcal{B}_{R}$, where $R$ is assumed to be large. We show in the next section that the problem is reduced to seeking solutions of the following boundary value problem:

$$
\left\{\begin{array}{rlll}
-\mu \Delta \mathbf{u}-(\lambda+\mu) \nabla \operatorname{div} \mathbf{u} & =\mathbf{0} & \text { in } \mathcal{B}_{R} \backslash \omega \\
\sigma(\mathbf{u}) \cdot \mathbf{n} & =\mathbf{G} & \text { on } \partial \omega \\
\frac{R(1+\nu)}{E} \sigma(\mathbf{u}) \cdot \mathbf{n}+\frac{R^{2}}{2}\left[\begin{array}{cc}
-\frac{\nu}{2(1-\nu)} & 0 \\
0 & \frac{1-\nu}{1-2 \nu}
\end{array}\right] \Delta_{\tau} \mathbf{u}+\mathbf{u} & =\mathbf{0} & \text { on } \partial \mathcal{B}_{R},
\end{array}\right.
$$

with $\mathbf{G} \in \mathrm{H}^{1 / 2}(\partial \omega), \omega$ is a $\mathcal{C}^{\infty}$ perturbation of the unit ball and $E, \nu$ the Young's modulus and Poisson's ratio linked to the Lamé coefficient by relation (2.9), while $\Delta_{\tau}$ denotes the Laplace-Beltrami operator on $\partial \Omega$. Let us recall the definition of the tangential differential operators: the tangential gradient on $\partial \Omega$ of $u \in \mathrm{H}^{2}(\Omega)$ is $\nabla_{\tau} u=\nabla u-\partial_{\mathbf{n}} u \mathbf{n}$, the tangential divergence $\operatorname{div}_{\tau}$ is its $L^{2}(\partial \Omega)$ adjoint and the Laplace-Beltrami operator is $\operatorname{div}_{\tau}\left(\nabla_{\tau} \cdot\right)$.

The non-standard condition on the outer ball in (1.4) is known as a Ventcel boundary condition (i.e. involving second order tangential derivatives of the displacement field). These conditions appear frequently as the result of an asymptotic analysis to derive impedance conditions for thin layer problems (see [6, 17, 22]). The first order approximation for the Laplace-Neumann transmission problem with a 
thin layer is

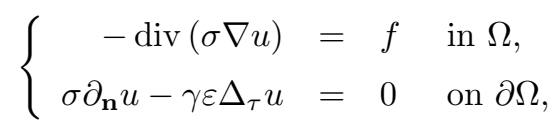

where $\sigma$ and $\gamma$ stand for the conductivity in the core and in the layer of thickness $\varepsilon$ respectively. It leads to the variational form

$$
\int_{\Omega} \sigma \nabla u \cdot \nabla v+\gamma \varepsilon \int_{\partial \Omega} \nabla_{\tau} u \cdot \nabla_{\tau} v=\int_{\Omega} f v .
$$

Since $\gamma>0$, this problem is, up to the constants, coercive on the Hilbert space $V=\left\{v \in \mathrm{H}^{1}(\Omega), v_{\mid \partial \Omega} \in \mathrm{H}^{1}(\partial \Omega)\right\}$ and therefore well posed. These coercive Ventcel boundary conditions also appear in the context of wall laws for rough boundaries (see [1, 2, 24]).

Due to the physical range of the parameters, problem (1.4) is not coercive. This difficulty also appears in other models arising from an asymptotic analysis. Let us give three examples in various applied fields. In electromagnetism, Delourme studied coaxial tubes coated by wires separated by a distance $\delta$. On a model problem with a simplified geometry, she obtained approximate transmission conditions (set on an interface replacing the layer of thickness $\delta$ ) of order one which are non-coercive Ventcel conditions [14,15. The same type of transmission conditions appear in the work 28] of Marigo and Pideri in the context of linear elasticity in dimension three, where the authors study the effects of inclusions of a second material under the assumptions that the defects are coplanar. Finally, in the context of wall laws for rough boundary, Bresch and Milisic obtained in [12] a second order wall law which is written in the form

$$
\left\{\begin{array}{rll}
-\Delta \mathcal{V}_{\varepsilon} & =C & \text { in } \Omega, \\
\mathcal{V}_{\varepsilon}-\varepsilon \beta\left(x_{1}, 0\right) \partial_{\mathbf{n}} \mathcal{V}_{\varepsilon}-\frac{\varepsilon^{2}}{2} \gamma\left(x_{1}, 0\right) \partial_{\tau}^{2} \mathcal{V}_{\varepsilon} u & =0 & \text { on } \Gamma^{0},
\end{array}\right.
$$

where $\beta$ and $\gamma$ are solutions of the two cells problem and have opposite signs.

In a first work on the scalar case [9, we studied the existence and uniqueness for the non-coercive Ventcel problem. In this paper we give the first results, to the best of our knowledge, in the vectorial case.

The main result of this paper is the following theorem, which ensures that problem (1.4) is well posed for $R$ large enough:

Theorem 1.1. There is a countable set of parameters $\mathcal{S}$ such that for any $\nu \notin \mathcal{S}$ we have the following results:

(1) Suppose $\omega=\mathcal{B}_{1}$. Then there is a bounded and at most countable set $\mathcal{R}_{\nu}$ such that for all $R \notin \mathcal{R}_{\nu}$ and for all $\mathbf{G} \in \mathrm{H}^{1 / 2}(\partial \omega)$, problem (1.4) admits a unique solution $\mathbf{u} \in \mathrm{H}^{2}\left(\mathcal{B}_{R} \backslash \omega\right)$.

(2) Suppose $\omega$ is a small perturbation of $\mathcal{B}_{1}$ in the following sense: $\omega=(\mathrm{Id}+$ $\boldsymbol{h}) \mathcal{B}_{1}$ with $\boldsymbol{h} \in \mathcal{C}^{\infty}$ and $\|\omega\|_{\mathrm{W}^{1, \infty}}<1$. Then there exist $\varepsilon_{\nu}$ and $R_{\nu}$ such that for all $R>R_{\nu}$, for all $\mathbf{G} \in \mathrm{H}^{1 / 2}(\partial \omega)$, and for all $\|\boldsymbol{h}\|_{\mathrm{W}^{1, \infty}} \leq \varepsilon_{\nu}$, problem (1.4) admits a unique solution $\mathbf{u} \in \mathrm{H}^{2}\left(\mathcal{B}_{R} \backslash \omega\right)$.

We show in Section 3 that Theorem 1.1 is a consequence of a careful analysis of another reduced problem on the boundary $\partial \mathcal{B}_{R}$ of the following type:

$$
\frac{1}{2}\left[\begin{array}{cc}
-\frac{\nu}{2(1-\nu)} & 0 \\
0 & \frac{1-\nu}{1-2 \nu}
\end{array}\right] \partial_{\theta}^{2} \varphi+\varphi+\Lambda_{R}(\varphi)=-\frac{R(1+\nu)}{E} \sigma\left(\mathbf{u}_{0}\right) \cdot \mathbf{n} .
$$


We point out that the Dirichlet-to-Neumann map $\Lambda_{R}$ (see (3.2) for the precise definition) has been studied as a pseudodifferential operator by Nakamura and Uhlmann in 33,34 , where the symbol in the pseudodifferential framework is written explicitly. Nevertheless the expression given there is not suitable for our study with a parameter $R$, for which we need more quantitative information.

In Section 2, we derive the artificial boundary condition (1.4). In Section 3, we show the existence of the solution of the boundary value problem in the sense of Theorem 1.1. The strategy of the proof consists in reducing the problem to equation (1.7), which can be analyzed after projection onto a family of finite subspaces of functions. An explicit study of the uniform solvability is then performed for the projected linear systems. We give an explicit form for the Dirichlet-to-Neumann map for the elasticity system in the particular geometric configuration where $\omega$ is a disk. Next, we extend the result when $\omega$ is close to a disk by a perturbation method. In Section 4, we present the proof of the main Proposition 3.2 stated in Section 3 . Finally, we present in Section 5 numerical illustrations of our results.

\section{Derivation of the ARTIFICIAL BOUNDARY CONDITION}

2.1. Singularities at infinity for the elasticity problem. We consider problem (1.3) set in the perturbed plane $\mathbf{H}_{\infty}=\mathbb{R}^{2} \backslash \bar{\omega}$, defining the profile. Let $R$ denote a positive real number and $\mathcal{B}_{R}$ be the ball of radius $R$ centered at the origin. To derive artificial boundary conditions for the linear elasticity on $\partial \mathcal{B}_{R}$ for large $R$, we need to know the precise behavior of the solution $\mathbf{v}_{\ell}$ of problem (1.3) at infinity. In the following, $L$ denotes the operator $L=\mu \Delta+(\lambda+\mu) \nabla$ div.

It is natural to introduce polar coordinates:

$$
\mathbf{u}(r, \theta)=u_{r}(r, \theta) \mathbf{e}_{r}+u_{\theta}(r, \theta) \mathbf{e}_{\theta},
$$

with $\mathbf{e}_{r}=\cos \theta \mathbf{e}_{1}+\sin \theta \mathbf{e}_{2}$ and $\mathbf{e}_{\theta}=-\sin \theta \mathbf{e}_{1}+\cos \theta \mathbf{e}_{2}$.

We recall the expression of the involved differential operators in the polar system

$$
\begin{aligned}
\Delta \mathbf{u}= & \left(\partial_{r}^{2} u_{r}+\frac{1}{r} \partial_{r} u_{r}-\frac{1}{r^{2}} u_{r}+\frac{1}{r^{2}} \partial_{\theta}^{2} u_{r}-\frac{2}{r^{2}} \partial_{\theta} u_{\theta}\right) \mathbf{e}_{r} \\
& +\left(\partial_{r}^{2} u_{\theta}+\frac{1}{r} \partial_{r} u_{\theta}-\frac{1}{r^{2}} u_{\theta}+\frac{1}{r^{2}} \partial_{\theta}^{2} u_{\theta}+\frac{2}{r^{2}} \partial_{\theta} u_{r}\right) \mathbf{e}_{\theta}, \\
\operatorname{div} \mathbf{u}= & \partial_{r} u_{r}+\frac{1}{r} u_{r}+\frac{1}{r} \partial_{\theta} u_{\theta}, \\
\nabla(\operatorname{div} \mathbf{u})= & \left(\partial_{r}^{2} u_{r}+\frac{1}{r} \partial_{r} u_{r}-\frac{1}{r^{2}} u_{r}-\frac{1}{r^{2}} \partial_{\theta} u_{\theta}+\frac{1}{r} \partial_{r \theta}^{2} u_{\theta}\right) \mathbf{e}_{r} \\
& +\left(\frac{1}{r} \partial_{r \theta}^{2} u_{r}+\frac{1}{r^{2}} \partial_{\theta} u_{r}+\frac{1}{r^{2}} \partial_{\theta}^{2} u_{\theta}\right) \mathbf{e}_{\theta} .
\end{aligned}
$$

Then the operator $L$ takes the form

$$
\begin{aligned}
& \left((\lambda+2 \mu)\left[\partial_{r}^{2} u_{r}+\frac{1}{r} \partial_{r} u_{r}-\frac{1}{r^{2}} u_{r}\right]+\frac{\mu}{r^{2}} \partial_{\theta}^{2} u_{r}-\frac{\lambda+3 \mu}{r^{2}} \partial_{\theta} u_{\theta}+\frac{\lambda+\mu}{r} \partial_{r \theta}^{2} u_{\theta}\right) \mathbf{e}_{r} \\
& +\left(\mu\left[\partial_{r}^{2} u_{\theta}+\frac{1}{r} \partial_{r} u_{\theta}-\frac{1}{r^{2}} u_{\theta}\right]+\frac{\lambda+2 \mu}{r^{2}} \partial_{\theta}^{2} u_{\theta}+\frac{\lambda+\mu}{r} \partial_{r \theta}^{2} u_{r}+\frac{\lambda+3 \mu}{r^{2}} \partial_{\theta} u_{r}\right) \mathbf{e}_{\theta},
\end{aligned}
$$


and the stress tensor is given by

$$
\sigma(\mathbf{u})=\left[\begin{array}{cc}
(\lambda+2 \mu) \partial_{r} u_{r}+\frac{\lambda}{r}\left(u_{r}+\partial_{\theta} u_{\theta}\right) & \mu\left(\frac{1}{r}\left(\partial_{\theta} u_{r}-u_{\theta}\right)+\partial_{r} u_{\theta}\right) \\
\mu\left(\frac{1}{r}\left(\partial_{\theta} u_{r}-u_{\theta}\right)+\partial_{r} u_{\theta}\right) & (\lambda+2 \mu) \frac{1}{r}\left(\partial_{\theta} u_{\theta}+u_{r}\right)+\lambda \partial_{r} u_{r}
\end{array}\right]
$$

Singularities of elliptic problems appear to be of tensorial form; see [13, 19, 32, and especially [20,21,26] for the elasticity system. Therefore, we seek solutions of $L \mathbf{u}=0$ under the form

$$
\mathbf{u}(r, \theta)=r^{k}\left[\begin{array}{l}
\phi_{r}(\theta) \\
\phi_{\theta}(\theta)
\end{array}\right]
$$

Consequently, using (2.1) and (2.2), we have in polar coordinates

$$
\begin{gathered}
L \mathbf{u}=r^{k-2}\left[\begin{array}{cc}
\mu \phi_{r}^{\prime \prime}+(\lambda+2 \mu)\left(k^{2}-1\right) \phi_{r}+[(\lambda+\mu) k-(\lambda+3 \mu)] \phi_{\theta}^{\prime} \\
(\lambda+2 \mu) \phi_{\theta}^{\prime \prime}+\mu\left(k^{2}-1\right) \phi_{\theta}+[(\lambda+\mu) k+(\lambda+3 \mu)] \phi_{r}^{\prime}
\end{array}\right] \\
\sigma(\mathbf{u})=r^{k-1}\left[\begin{array}{cc}
\lambda \phi_{\theta}^{\prime}+((\lambda+2 \mu) k+\lambda) \phi_{r} & \mu\left(\phi_{r}^{\prime}+(k-1) \phi_{\theta}\right) \\
\mu\left(\phi_{r}^{\prime}+(k-1) \phi_{\theta}\right) & (\lambda+2 \mu) \phi_{\theta}^{\prime}+(\lambda k+(\lambda+2 \mu)) \phi_{r}
\end{array}\right]
\end{gathered}
$$

Using (2.4), we reduce the second order system $L \mathbf{u}=0$ into a bigger system of first order. Introducing $\psi_{r}=\phi_{r}^{\prime}, \psi_{\theta}=\phi_{\theta}^{\prime}$, and $\mathbf{U}=\left(\phi_{r}, \phi_{\theta}, \psi_{r}, \psi_{\theta}\right)^{\top}$, we get the matrix formulation

$$
\mathbf{U}^{\prime}=A \mathbf{U}
$$

with

$$
A=\left[\begin{array}{cccc}
0 & 0 & 1 & 0 \\
0 & 0 & 0 & 1 \\
\frac{(\lambda+2 \mu)\left(1-k^{2}\right)}{\mu} & 0 & 0 & \frac{(\lambda+3 \mu)-(\lambda+\mu) k}{\mu} \\
0 & \frac{\mu\left(1-k^{2}\right)}{\lambda+2 \mu} & -\frac{(\lambda+3 \mu)+(\lambda+\mu) k}{\lambda+2 \mu} & 0
\end{array}\right]
$$

The eigenvalues of $A$ are $\pm i(k \pm 1)$. Hence, the polar functions $\phi_{r}, \phi_{\theta}$ belong to the space generated by $\cos ((k \pm 1) \theta), \sin ((k \pm 1) \theta)$. The continuity for $\theta=0, \theta=2 \pi$ requires $k$ to be an integer.

The integer $k$ being fixed, we look for coefficients $A_{r}, B_{r}, C_{r}, D_{r}, A_{\theta}, B_{\theta}, C_{\theta}, D_{\theta}$, so that

$$
\begin{aligned}
& \phi_{r}(\theta)=A_{r} \cos ((k-1) \theta)+B_{r} \sin ((k-1) \theta)+C_{r} \cos ((k+1) \theta)+D_{r} \sin ((k+1) \theta), \\
& \phi_{\theta}(\theta)=A_{\theta} \cos ((k-1) \theta)+B_{\theta} \sin ((k-1) \theta)+C_{\theta} \cos ((k+1) \theta)+D_{\theta} \sin ((k+1) \theta) .
\end{aligned}
$$

Writing $L \mathbf{u}=0$, we get

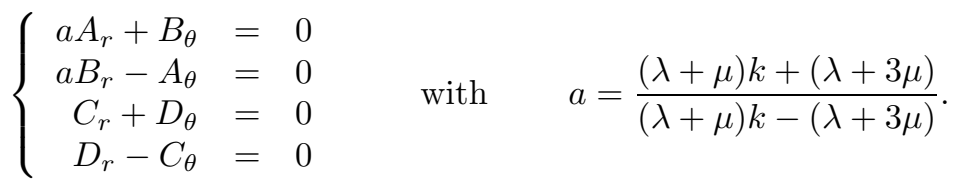

Consequently, the functions $\phi_{r}$ and $\phi_{\theta}$ satisfy

$$
\begin{gathered}
\phi_{r}(\theta)=A_{r} \cos ((k-1) \theta)+B_{r} \sin ((k-1) \theta)+C_{r} \cos ((k+1) \theta)+D_{r} \sin ((k+1) \theta), \\
\phi_{\theta}(\theta)=a B_{r} \cos ((k-1) \theta)-a A_{r} \sin ((k-1) \theta)+D_{r} \cos ((k+1) \theta)-C_{r} \sin ((k+1) \theta) .
\end{gathered}
$$


Remark 2.1. It can be shown that these singular functions describe the behavior at infinity of the solutions of the elasticity system in the plane. The solution $\mathbf{v}_{\ell}$ of (1.3) satisfies for any $N>0$

$$
\mathbf{v}_{\ell}(x)=\sum_{-N<k<0} \mathbf{v}_{\ell, k}(x)+\mathcal{O}_{|x| \rightarrow \infty}\left(|x|^{-N}\right),
$$

where $\mathbf{v}_{\ell, k}$ has the separated variables structure as in (2.3). The sum is extended to negative integers since $\mathbf{v}_{\ell}$ vanishes at infinity. Let us mention that such an expansion still holds for the derivatives of $\mathbf{v}_{\ell}$ of any order.

2.2. Artificial boundary condition on $\partial \mathcal{B}_{R}$. To approximate problem (1.3), we introduce the bounded domain $\mathbf{H}_{R}=\mathbf{H}_{\infty} \cap \mathcal{B}_{R}$, where $\mathcal{B}_{R}$ is the ball of radius $R$ centered at the origin. We are looking for a boundary condition to impose on the artificial boundary $\partial \mathcal{B}_{R}$. Since $\mathbf{v}_{\ell}$ tends to 0 at infinity, a first (naive) choice consists in setting a homogeneous Dirichlet condition on $\partial \mathcal{B}_{R}$. However, thanks to expansion (2.7), the resulting error is of order $\mathcal{O}\left(R^{-1}\right)$, which is rather poor. To improve this approximation accuracy, we seek a boundary condition which is satisfied by the leading term in (2.7) so that the error becomes of order $\mathcal{O}\left(R^{-2}\right)$.

More precisely, we find a linear relation between displacement and traction on the artificial boundary $\partial \mathcal{B}_{R}$. If $k=-1$, the relation $L \mathbf{u}=0$ reads

$$
\left\{\begin{aligned}
\mu \phi_{r}^{\prime \prime}-2(\lambda+2 \mu) \phi_{\theta}^{\prime} & =0 \\
(\lambda+2 \mu) \phi_{\theta}^{\prime \prime}+2 \mu \phi_{r}^{\prime} & =0
\end{aligned}\right.
$$

To determine artificial boundary conditions on $\partial \mathcal{B}_{R}$, we consider $\sigma(\mathbf{u}) \cdot \mathbf{n}$ and notice that $\mathbf{n}=\mathbf{e}_{r}$ on $\partial \mathcal{B}_{R}$. Using (2.5) and (2.8), we get for $k=-1$ :

$$
\begin{aligned}
\sigma(\mathbf{u}) \cdot \mathbf{e}_{r} & =r^{-2}\left[\begin{array}{c}
\lambda \phi_{\theta}^{\prime} \\
\mu \phi_{r}^{\prime}
\end{array}\right]-2 \mu r^{-2}\left[\begin{array}{l}
\phi_{r} \\
\phi_{\theta}
\end{array}\right] \\
& =r^{-2}\left[\begin{array}{cc}
\frac{\lambda \mu}{2(\lambda+2 \mu)} & 0 \\
0 & -\frac{\lambda+2 \mu}{2}
\end{array}\right]\left[\begin{array}{c}
\phi_{r}^{\prime \prime} \\
\phi_{\theta}^{\prime \prime}
\end{array}\right]-2 \mu r^{-2}\left[\begin{array}{c}
\phi_{r} \\
\phi_{\theta}
\end{array}\right]
\end{aligned}
$$

Consequently

$$
\sigma(\mathbf{u}) \cdot \mathbf{n}+\frac{R}{2}\left[\begin{array}{cc}
-\frac{\lambda \mu}{\lambda+2 \mu} & 0 \\
0 & \lambda+2 \mu
\end{array}\right] \Delta_{\tau} \mathbf{u}+\frac{2 \mu}{R} \mathbf{u}=0
$$

Lamé's coefficients are linked to the physical parameters (Young's modulus and Poisson's ratio) through the following relations:

$$
\left\{\begin{aligned}
\lambda & =\frac{\nu E}{(1+\nu)(1-2 \nu)} \\
\mu & =\frac{E}{2(1+\nu)}
\end{aligned} \quad \text { with } \quad E>0,-1<\nu<0.5\right.
$$

so that this boundary condition of Ventcel's type rewrites on $\partial \mathcal{B}_{R}$ :

$$
\sigma(\mathbf{u}) \cdot \mathbf{n}+\frac{R E}{2(1+\nu)}\left[\begin{array}{cc}
\frac{-\nu}{2(1-\nu)} & 0 \\
0 & \frac{1-\nu}{1-2 \nu}
\end{array}\right] \Delta_{\tau} \mathbf{u}+\frac{E}{R(1+\nu)} \mathbf{u}=0 .
$$


We notice that

$$
\frac{1-\nu}{(1+\nu)(1-2 \nu)}>0 \quad \text { and } \begin{cases}\frac{-\nu}{2\left(1-\nu^{2}\right)}<0 & \text { if } \quad \nu \in(0,0.5), \\ \frac{-\nu}{2\left(1-\nu^{2}\right)}>0 & \text { if } \quad \nu \in(-1,0) .\end{cases}
$$

We finally get the following boundary value problem: Given $\mathbf{G} \in \mathrm{H}^{1 / 2}(\partial \omega)$, find $\mathbf{u} \in \mathrm{H}^{2}\left(\mathcal{B}_{R} \backslash \omega\right)$ such that

$$
\left\{\begin{aligned}
-\mu \Delta \mathbf{u}-(\lambda+\mu) \nabla \operatorname{div} \mathbf{u} & =\mathbf{0} & \text { in } \mathcal{B}_{R} \backslash \omega \\
\sigma(\mathbf{u}) \cdot \mathbf{n} & =\mathbf{G} & \text { on } \partial \omega \\
\frac{R(1+\nu)}{E} \sigma(\mathbf{u}) \cdot \mathbf{n}+\frac{R^{2}}{2}\left[\begin{array}{cc}
-\frac{\nu}{2(1-\nu)} & 0 \\
0 & \frac{1-\nu}{1-2 \nu}
\end{array}\right] \Delta_{\tau} \mathbf{u}+\mathbf{u} & =\mathbf{0} & \text { on } \partial \mathcal{B}_{R} .
\end{aligned}\right.
$$

This last equation is written on the polar basis $\left(\mathbf{e}_{r}, \mathbf{e}_{\theta}\right)$, i.e. $\mathbf{u}=\left(u_{r}, u_{\theta}\right)$. Whatever the sign of the parameter $\nu \in(-1,0.5)$, the obtained approximate boundary condition leads to a non-coercive weak formulation. A similar problem has been investigated in [9] for the scalar Laplace equation.

\section{Solvability of the Equations}

3.1. The strategy. We make a change of the unknown by splitting the unknown function $\mathbf{u} \in \mathrm{H}^{2}\left(\mathcal{B}_{R} \backslash \omega\right)$ of problem (2.10) into the sum

$$
\mathbf{u}=\mathbf{u}_{0}+\mathbf{v}
$$

where $\mathbf{u}_{0} \in \mathrm{H}^{2}\left(\mathcal{B}_{R} \backslash \omega\right)$ lifts the traction $\mathbf{G} \in \mathrm{H}^{1 / 2}(\partial \omega)$, i.e. satisfies the following problem with a Dirichlet condition on the artificial boundary:

$$
\left\{\begin{aligned}
-\mu \Delta \mathbf{u}_{0}-(\lambda+\mu) \nabla \operatorname{div} \mathbf{u}_{0} & =\mathbf{0} & & \text { in } \mathcal{B}_{R} \backslash \omega \\
\sigma\left(\mathbf{u}_{0}\right) \cdot \mathbf{n} & =\mathbf{G} & & \text { on } \partial \omega \\
\mathbf{u}_{0} & =0 & & \text { on } \partial \mathcal{B}_{R} .
\end{aligned}\right.
$$

We define the Dirichlet-to-Neumann map $\Lambda_{R}$ for all $s>0$ by

$$
\begin{array}{ccc}
\Lambda_{R}: \mathrm{H}^{s+1 / 2}\left(\partial \mathcal{B}_{R}\right) & \rightarrow & \mathrm{H}^{s-1 / 2}\left(\partial \mathcal{B}_{R}\right) \\
\boldsymbol{\varphi} & \mapsto & \frac{R(1+\nu)}{E} \sigma(\mathbf{v}) \cdot \mathbf{n},
\end{array}
$$

where $\mathbf{v}$ is the solution in $\mathrm{H}^{s+1}\left(\mathcal{B}_{R} \backslash \omega\right)$ of

$$
\left\{\begin{array}{rlll}
-\mu \Delta \mathbf{v}-(\lambda+\mu) \nabla \operatorname{div} \mathbf{v} & =\mathbf{0} & & \text { in } \mathcal{B}_{R} \backslash \omega \\
\sigma(\mathbf{v}) \cdot \mathbf{n} & =\mathbf{0} & & \text { on } \partial \omega \\
\mathbf{v} & =\boldsymbol{\varphi} & & \text { on } \partial \mathcal{B}_{R}
\end{array}\right.
$$

Let $\varphi$ be the solution in $\mathrm{H}^{3 / 2}\left(\partial \mathcal{B}_{R}\right)$ of

$$
\frac{R(1+\nu)}{E} \sigma\left(\mathbf{u}_{0}\right) \cdot \mathbf{n}+\frac{1}{2}\left[\begin{array}{cc}
-\frac{\nu}{2(1-\nu)} & 0 \\
0 & \frac{1-\nu}{1-2 \nu}
\end{array}\right] \partial_{\theta}^{2} \boldsymbol{\varphi}+\boldsymbol{\varphi}+\Lambda_{R}(\boldsymbol{\varphi})=0 .
$$

Then $\mathbf{u}$ is the solution in $\mathrm{H}^{2}\left(\mathcal{B}_{R} \backslash \omega\right)$ of $(2.10)$ if and only if $\mathbf{u}_{0}$ is the solution in $\mathrm{H}^{2}\left(\mathcal{B}_{R} \backslash \omega\right)$ of (3.1) and $\mathbf{v}$ is the solution in $\mathrm{H}^{2}\left(\mathcal{B}_{R} \backslash \omega\right)$ of (3.3) with $\varphi$ the solution in $\mathrm{H}^{3 / 2}\left(\partial \mathcal{B}_{R}\right)$ of (3.4). 
We aim at proving that problem (3.4) is well posed for any $R>0$ except in a bounded and countable set. We therefore have to reduce the problem to the following boundary problem of unknown $\varphi \in \mathrm{H}^{3 / 2}\left(\partial \mathcal{B}_{R}\right)$, and where $\mathbf{u}_{0}$ is given:

$$
\frac{1}{2}\left[\begin{array}{cc}
-\frac{\nu}{2(1-\nu)} & 0 \\
0 & \frac{1-\nu}{1-2 \nu}
\end{array}\right] \partial_{\theta}^{2} \boldsymbol{\varphi}+\boldsymbol{\varphi}+\Lambda_{R}(\boldsymbol{\varphi})=-\frac{R(1+\nu)}{E} \sigma\left(\mathbf{u}_{0}\right) \cdot \mathbf{n} .
$$

In order to solve it, we shall give a concrete expression of $\Lambda_{R}$ and work in polar coordinates.

Remark 3.1. A careful analysis of the above strategy shows that we have the following higher regularity properties for the unknown functions: $\mathbf{u}_{0} \in \mathrm{H}^{2}\left(\mathcal{B}_{R} \backslash \omega\right)$ as stated, but $\varphi \in \mathrm{H}^{5 / 2}\left(\partial \mathcal{B}_{R}\right)$ and $\mathbf{v} \in \mathrm{H}^{3}\left(\mathcal{B}_{R} \backslash \omega\right)$ by elliptic regularity, since (as a direct consequence of Proposition 3.2 below) $\Lambda_{R}$ is in fact an operator of order 1. Note also that the decoupling into two problems (3.1) on the one hand and (3.3) - (3.4) on the other hand was necessary in order to be able to introduce $\Lambda_{R}$ as an operator (i.e. an unbounded linear application).

3.2. A decoupled system when the inclusion is a disk. We consider the case where $\omega$ is the disk $\mathcal{B}_{1}$ of radius 1 centered at the origin. Boundary problem (3.3) defining the Dirichlet-to-Neumann map is then set in a ring. It is natural to write the Dirichlet datum $\varphi$ as a Fourier series:

$$
\boldsymbol{\varphi}=\left[\begin{array}{c}
\varphi_{0}^{r} \\
\varphi_{0}^{\theta}
\end{array}\right]+\sum_{n \geq 1}\left[\begin{array}{c}
\varphi_{n}^{r} \\
\varphi_{n}^{\theta}
\end{array}\right] \cos n \theta+\sum_{n \geq 1}\left[\begin{array}{c}
\psi_{n}^{r} \\
\psi_{n}^{\theta}
\end{array}\right] \sin n \theta
$$

We will show in Propositions 3.2 and 3.3 below that the Dirichlet-to-Neumann map takes the form

$$
\Lambda_{R}(\boldsymbol{\varphi})=\Lambda_{R}^{0}(\boldsymbol{\varphi})+\mathcal{R}(\boldsymbol{\varphi})
$$

where the principal part $\Lambda_{R}^{0}$ is defined in polar coordinates by

$$
\begin{aligned}
\Lambda_{R}^{0}(\boldsymbol{\varphi})=\frac{1-\gamma}{\gamma}\left[\begin{array}{c}
\varphi_{0}^{r} \\
0
\end{array}\right]+\frac{1}{1+\gamma} \sum_{n \geq 1}\left[\begin{array}{c}
(n-\gamma) \varphi_{n}^{r}+(1-n \gamma) \psi_{n}^{\theta} \\
(n \gamma-1) \psi_{n}^{r}+(n-\gamma) \varphi_{n}^{\theta}
\end{array}\right] \cos n \theta \\
+\frac{1}{1+\gamma} \sum_{n \geq 1}\left[\begin{array}{c}
(n-\gamma) \psi_{n}^{r}+(n \gamma-1) \varphi_{n}^{\theta} \\
(1-n \gamma) \varphi_{n}^{r}+(n-\gamma) \psi_{n}^{\theta}
\end{array}\right] \sin n \theta
\end{aligned}
$$

where

$$
\gamma=\frac{1-2 \nu}{2(1-\nu)}
$$

and the remainder $\mathcal{R}(\varphi)$ is controlled. In the following proposition, we reformulate problem (3.5) in terms of the unknown Fourier coefficients $\varphi_{n}^{r}, \varphi_{n}^{\theta}, \psi_{n}^{r}$, and $\psi_{n}^{\theta}$.

Proposition 3.2. Let $\boldsymbol{\varphi} \in H^{3 / 2}\left(\partial \mathcal{B}_{R}\right)$. For all $n \geq 1$, let $\boldsymbol{\Phi}_{n}=\left(\varphi_{n}^{r}, \psi_{n}^{r}, \varphi_{n}^{\theta}, \psi_{n}^{\theta}\right)^{\top}$, corresponding to the $n$-th coordinates of $\boldsymbol{\varphi}$ in (3.6), and let $\mathbf{f}_{n, R}$ be the 4-uplet of the decomposition of $-\frac{R(1+\nu)}{E} \sigma\left(\mathbf{u}_{0}\right) \cdot \mathbf{n}$ with respect to the same basis:

$$
-\frac{R(1+\nu)}{E} \sigma\left(\mathbf{u}_{0}\right) \cdot \mathbf{n}=\left[\begin{array}{l}
\left(\mathbf{f}_{0, R}\right)_{1} \\
\left(\mathbf{f}_{0, R}\right)_{2}
\end{array}\right]+\sum_{n \geq 1}\left[\begin{array}{l}
\left(\mathbf{f}_{n, R}\right)_{1} \\
\left(\mathbf{f}_{n, R}\right)_{3}
\end{array}\right] \cos n \theta+\sum_{n \geq 1}\left[\begin{array}{l}
\left(\mathbf{f}_{n, R}\right)_{2} \\
\left(\mathbf{f}_{n, R}\right)_{4}
\end{array}\right] \sin n \theta .
$$

Similarly, let $\boldsymbol{\Phi}_{0}=\left(\varphi_{0}^{r}, \varphi_{0}^{\theta}\right)^{\top}$ correspond to the 0-th coordinates of $\boldsymbol{\varphi}$ in (3.6), respectively $\mathbf{f}_{0, R}$ be the 2 -uplet of the decomposition of $-\frac{R(1+\nu)}{E} \sigma\left(\mathbf{u}_{0}\right) \cdot \mathbf{n}$ with respect 
to the last basis. Then equation (3.5) reads

$$
P_{n} \boldsymbol{\Phi}_{n}+\mathcal{R}_{n, R} \boldsymbol{\Phi}_{n}=\mathbf{f}_{n, R},
$$

where $P_{n}$ is the matrix given by

for $n \geq 1$,

$$
\begin{aligned}
P_{n}=\frac{-n^{2}}{4}\left[\begin{array}{cccc}
1-2 \gamma & 0 & 0 & 0 \\
0 & 1-2 \gamma & 0 & 0 \\
0 & 0 & \frac{1}{\gamma} & 0 \\
0 & 0 & 0 & \frac{1}{\gamma}
\end{array}\right]+\operatorname{Id}_{4} \\
+\frac{1}{1+\gamma}\left[\begin{array}{cccc}
n-\gamma & 0 & 0 & 1-n \gamma \\
0 & n-\gamma & n \gamma-1 & 0 \\
0 & n \gamma-1 & n-\gamma & 0 \\
1-n \gamma & 0 & 0 & n-\gamma
\end{array}\right],
\end{aligned}
$$

for $n=0$,

$$
P_{0}=0+\operatorname{Id}_{2}+\left[\begin{array}{cc}
\frac{1-\gamma}{\gamma} & 0 \\
0 & 0
\end{array}\right]=\left[\begin{array}{cc}
\frac{1}{\gamma} & 0 \\
0 & 1
\end{array}\right],
$$

and the remainder $\mathcal{R}_{n, R}$ is controlled uniformly in $n \in \mathbb{N}$ for $R$ large: there exist $R_{0}>0$ and a constant $C$ (independent of $n$ and $R$ ) such that for any $n$ and $R>R_{0}$, we have

$$
\left\{\begin{array}{l}
\left\|\mathcal{R}_{n, R}\right\|_{\infty} \leq C n^{2} R^{-2 n+2} \quad \text { if } \quad n \geq 2 \\
\left\|\mathcal{R}_{1, R}\right\|_{\infty} \leq C R^{-4} \\
\left\|\mathcal{R}_{0, R}\right\|_{\infty} \leq C R^{-2}
\end{array}\right.
$$

Proof. The proof of this fundamental result is postponed to Section 4.

Note that (3.8) is a consequence of the expression of $\Lambda_{R}^{0}$ in terms of Fourier coefficients. Using decomposition (3.6) and denoting $\boldsymbol{\Phi}_{n}=\left(\varphi_{n}^{r}, \psi_{n}^{r}, \varphi_{n}^{\theta}, \psi_{n}^{\theta}\right)^{\top}$, this relation reads

$$
\Lambda_{R}^{0}(\boldsymbol{\varphi})=P_{0}^{0}\left[\begin{array}{l}
\varphi_{0}^{r} \\
\varphi_{0}^{\theta}
\end{array}\right]+\sum_{n \geq 1}\left[\begin{array}{cccc}
\cos n \theta & \sin n \theta & 0 & 0 \\
0 & 0 & \cos n \theta & \sin n \theta
\end{array}\right] P_{n}^{0} \boldsymbol{\Phi}_{n}
$$

with

$$
P_{n}^{0}=\frac{1}{1+\gamma}\left[\begin{array}{cccc}
n-\gamma & 0 & 0 & 1-n \gamma \\
0 & n-\gamma & n \gamma-1 & 0 \\
0 & n \gamma-1 & n-\gamma & 0 \\
1-n \gamma & 0 & 0 & n-\gamma
\end{array}\right] \quad \text { when } n \geq 1
$$

and

$$
P_{0}^{0}=\left[\begin{array}{cc}
\frac{1-\gamma}{\gamma} & 0 \\
0 & 0
\end{array}\right] \quad \text { when } n=0 .
$$

These matrices have to be considered as a definition of operator $\Lambda_{R}^{0}$ in Fourier modes, and $\Lambda_{R}$ can be written in the equivalent usual form (3.8) by summing up the Fourier modes. Now we deal with the remainder term in (3.7):

$$
\mathcal{R}=\Lambda_{R}-\Lambda_{R}^{0} .
$$

Estimates (3.10) directly imply by summation in Fourier modes the following result: 
Proposition 3.3. For all $s \in \mathbb{R}$, there exists $C_{s}>0$ such that for any $\varphi \in$ $\mathcal{C}^{\infty}\left(\partial B_{R}\right)$ and $R>R_{0}$,

$$
\|\mathcal{R}(\varphi)\|_{\mathrm{H}^{s}\left(\partial \mathcal{B}_{R}\right)} \leq C_{-s} R^{-2}\|\varphi\|_{\mathrm{H}^{-s}\left(\partial \mathcal{B}_{R}\right)} .
$$

In particular, we have by natural extension that for all $\varphi \in \mathrm{H}^{1 / 2}\left(\partial \mathcal{B}_{R}\right)$,

$$
\|\mathcal{R}(\varphi)\|_{\mathrm{H}^{-1 / 2}\left(\partial \mathcal{B}_{R}\right)} \leq C_{1 / 2} R^{-2}\|\varphi\|_{\mathrm{H}^{1 / 2}\left(\partial \mathcal{B}_{R}\right)} .
$$

3.3. Well-posedness for the disk case. As a direct consequence of Propositions 3.2 and 3.3 , we get, in the case where $\omega$ is the disk $\mathcal{B}_{1}$, the following result of solvability for equation (3.5), and therefore for system (2.10). The expected regularity for each problem is the one stated in Subsection 3.1 when we developed our strategy: $\mathbf{u} \in \mathrm{H}^{2}\left(\mathcal{B}_{R} \backslash \omega\right), \boldsymbol{\varphi} \in \mathrm{H}^{3 / 2}\left(\partial \mathcal{B}_{R}\right)$ and $\mathbf{v} \in \mathrm{H}^{2}\left(\mathcal{B}_{R} \backslash \omega\right)$.

Proposition 3.4. Let us recall that $\gamma=\frac{1-2 \nu}{2(1-\nu)}$. We have

(1) The matrix $P_{n}$ is generically (with respect to $\gamma$ ) invertible for all $n$, which means that for each $\gamma \notin \mathcal{S}$, where $\mathcal{S}$ is an at most countable set of physical parameters, $P_{n}$ is invertible with norm of the inverse uniformly bounded in $n$.

(2) For all fixed $\gamma \notin \mathcal{S}$, there exists $R_{\gamma}$ such that equation (3.5) admits a unique solution $\varphi$ for all $R \geq R_{\gamma}$.

(3) For all $\gamma \notin \mathcal{S}$, there exists $R_{\gamma}$ such that system (2.10) admits a unique solution $\mathbf{u}$ for all $R \geq R_{\gamma}$.

(4) For all $\gamma \notin \mathcal{S}$, there exists a bounded and at most countable set $\mathcal{R}_{\gamma}$ such that system (2.10) admits a unique solution $\mathbf{u}$ for all $R \notin \mathcal{R}_{\gamma}$.

Remark 3.5. System (2.10) is expressed in terms of the parameter $\nu$. But since $\nu \mapsto \gamma=\frac{1-2 \nu}{2(1-\nu)}$ is a strictly decreasing function of $\nu$, it is equivalent to avoiding a countable set in $\nu$ or $\gamma$ variables. It is more convenient to make the computations with parameter $\gamma$, so we express the forbidden set in the $\gamma$-variable.

Proof. First we notice that the range of values of $\gamma$ is $(0,3 / 4)$ as $\nu \in(-1,1 / 2)$ since, as already mentioned, the function $\nu \mapsto \gamma=\frac{1-2 \nu}{2(1-\nu)}$ is decreasing on $(-1,1 / 2)$.

Let us deal with point (11). First, we observe that $P_{0}$ is actually invertible. For each fixed $n \geq 1$, we look at the full determinant of $P_{n}$ and show that only a finite number of values of $\gamma$ is forbidden. We can rewrite

$$
P_{n}=\left[\begin{array}{cccc}
A_{1} & 0 & 0 & B_{1} \\
0 & A_{1} & -B_{1} & 0 \\
0 & -B_{1} & A_{2} & 0 \\
B_{1} & 0 & 0 & A_{2}
\end{array}\right]
$$

with

$$
\begin{aligned}
& A_{1}=-\frac{n^{2}}{4}(1-2 \gamma)+1+\frac{n-\gamma}{1+\gamma}=\frac{n^{2}\left(1-\gamma+2 \gamma^{2}\right)+4 n+4}{4(1+\gamma)} \\
& A_{2}=\frac{-n^{2}}{4 \gamma}+1+\frac{n-\gamma}{1+\gamma}=\frac{-n^{2}(1+\gamma)+4 n \gamma+4 \gamma}{4 \gamma(1+\gamma)} \\
& B_{1}=\frac{1-n \gamma}{1+\gamma}
\end{aligned}
$$


Then, we compute $\operatorname{det} P_{n}$ and obtain

$$
\operatorname{det} P_{n}=\left(A_{1} A_{2}-B_{1}^{2}\right)^{2}=\frac{n^{2}}{16 \gamma^{2}(1+\gamma)^{2}}\left(\sum_{j=0}^{3} n^{j} P_{j}(\gamma)\right)^{2}
$$

with

$$
\begin{aligned}
& P_{0}(\gamma)=-32 \gamma, \\
& P_{1}(\gamma)=4\left(1-3 \gamma+2 \gamma^{2}\right)=4(1-2 \gamma)(1-\gamma), \\
& P_{2}(\gamma)=4\left(1+\gamma-2 \gamma^{2}\right)=4(1+2 \gamma)(1-\gamma), \\
& P_{3}(\gamma)=-1+\gamma+2 \gamma^{2}=(-1+2 \gamma)(1+\gamma) .
\end{aligned}
$$

Let $\Delta_{n}(\gamma):=16 \gamma^{2}(1+\gamma)^{2} \operatorname{det} P_{n}$. The expanded expression of $\Delta_{n}$ in terms of powers of $n$ is

$\Delta_{n}(\gamma)=n^{2}\left(\left(-1+\gamma+2 \gamma^{2}\right) n^{3}+4\left(1+\gamma-2 \gamma^{2}\right) n^{2}+4\left(1-3 \gamma+2 \gamma^{2}\right) n-32 \gamma\right)^{2}$.

We notice that $\Delta_{n}(\gamma)$ is a polynomial function in both $n$ and $\gamma$ of order at most 4 in $\gamma$. We want to check that $\Delta_{n}$ is not identically zero, and for this we look first at the value at $\gamma=0$ for $n$ fixed:

$$
\Delta_{n}(0)=n^{4}\left(-n^{2}+4 n+4\right)^{2} .
$$

It is clear that $\Delta_{n}$ is never identically zero, and therefore is a polynomial with respect to the variable $\gamma$ with at most 4 roots in the range $(0,3 / 4)$. We denote this set by $\mathcal{S}_{n}$ and set $\mathcal{S}=\left\{\frac{1}{2}\right\} \cup \bigcup_{n \in \mathbb{N}^{*}} \mathcal{S}_{n}$. Then $\mathcal{S}$ is at most countable. The real number $1 / 2$ should be avoided to make sure that the limit matrix appearing in the next paragraph is invertible.

Now for each $\gamma$ fixed in the complementary $\mathcal{S}^{c}$ of $\mathcal{S}$, we notice that the matrix $P_{n}$ is equivalent (in the asymptotic sense $n \rightarrow \infty$ ) to the matrix

$$
-\frac{n^{2}}{4}\left[\begin{array}{cccc}
1-2 \gamma & 0 & 0 & 0 \\
0 & 1-2 \gamma & 0 & 0 \\
0 & 0 & \frac{1}{\gamma} & 0 \\
0 & 0 & 0 & \frac{1}{\gamma}
\end{array}\right] .
$$

Recall that $\gamma \in \mathcal{S}^{c}$ (thus $\gamma \neq 1 / 2$ ) the previous matrix is thus invertible and implies that there exists $n_{\gamma}$ such that for all $n>n_{\gamma}, P_{n}$ also is invertible with inverse uniformly bounded with respect to $n$. For each integer $n$ in the finite set $\left\{1,2, \cdots, n_{\gamma}\right\}, P_{n}$ is again invertible since $\gamma \notin \mathcal{S}$, and the norm of the inverse can be bounded uniformly since there is only a finite number of values. At the end, we get that the norm of the inverse of $P_{n}$ can be bounded uniformly in $n \in \mathbb{N}$. This is the result of uniform boundedness of point (11).

For point (2), we first fix $\gamma \notin \mathcal{S}$. For any $n \geq 0$, we notice that the term $\mathcal{R}_{n, R}$ has a norm going to 0 uniformly in $n$ when $R$ goes to infinity (recall that this term also depends on $\gamma$ ), as mentioned in (3.10). This means that, according to point (1), there exists $R_{\gamma}$ such that for all $R>R_{\gamma}$, the matrix $P_{n}+\mathcal{R}_{n, R}$ is invertible for all $n \in \mathbb{N}$ with norm of this inverse uniformly bounded with respect to $n$. At the end, we have been able to solve the full problem of (3.5) mode by mode with a control (uniform in $n$ ) of the norm of the inverse. This gives the result.

For point (3), we just use the reduction at the beginning of Section 3 allowing us to reduce the problem on the boundary, and we get the result. 
For point (4), we fix $\gamma \notin \mathcal{S}$ and look at the problem mode by mode. For fixed $n \geq 0$, we can notice that for $M$ sufficiently large (for example $M>\max \{2 n-2,4\}$ ), the determinant of the matrix $R^{M} P_{n}+R^{M} \mathcal{R}_{n, R}$ built in the proof is a non-zero polynomial in $R$. This implies that for each $n$, only a finite number of radii give rise to the non-solvable equation (3.9). Again this means that for $R \notin \mathcal{R}_{\gamma}$, where $\mathcal{R}_{\gamma}$ is an at most countable set of radii, the problem $P_{n} \boldsymbol{\Phi}_{n}+\mathcal{R}_{n, R} \boldsymbol{\Phi}_{n}=\mathbf{f}_{n, R}$ is solvable for all $n \geq 0$, with norm of this inverse of $P_{n}+\mathcal{R}_{n, R}$ uniformly bounded with respect to $n$ (recall that $\mathbf{f}_{n, R}$ was defined in Proposition [3.2). As before we get that for $R \notin \mathcal{R}_{\gamma}$, problem (3.5) is solvable, and so is problem (2.10). Notice that point (2) implies that for each $\gamma \notin \mathcal{S}, \mathcal{R}_{\gamma}$ is a bounded set. The proof is completed.

3.4. A perturbation result for quasicircular inclusions. We aim at extending the previous result obtained for $\omega=\mathcal{B}_{1}$ to domains $\omega$ close to a ball. Now, applying point (3) of Proposition 3.4. we consider a real $R>1$ such that the system (2.10) admits a unique solution $\mathbf{u}$ on the domain $\mathcal{B}_{R} \backslash \mathcal{B}_{1}$ as soon as $\gamma \notin \mathcal{S}$. Consider a domain $\omega$ close to $\mathcal{B}_{1}$ and ask if system (2.10) admits a unique solution $\mathbf{u}$ on the domain $\mathcal{B}_{R} \backslash \omega$ for the same set of parameters $\mathcal{S}$. Our aim is to prove that the perturbed Dirichlet-to-Neumann map depends continuously (as an operator) on smooth perturbations of the domain.

We adapt directly [9, Theorem 3.1] and consider a $\mathcal{C}^{\infty}$ vector field $\boldsymbol{h}$ supported in $\mathcal{B}_{\rho}$ for $\rho \in(1, R)$ and the application $\mathrm{T}_{\boldsymbol{h}}: \mathbb{R}^{2} \rightarrow \mathbb{R}^{2}$ defined by $\mathrm{T}_{\boldsymbol{h}}=\mathrm{Id}_{\mathbb{R}^{2}}+\boldsymbol{h}$. Clearly, $\mathrm{T}_{\boldsymbol{h}}$ is a diffeomorphism when the norm of $\boldsymbol{h}$ is small; then the perturbed domain $\mathcal{B}_{R} \backslash \omega_{\boldsymbol{h}}$, with $\omega_{\boldsymbol{h}}=\mathrm{T}_{\boldsymbol{h}}(\omega)$, is just $\mathrm{T}_{\boldsymbol{h}}\left(\mathcal{B}_{R} \backslash \omega\right)$.

For any $s \geq 0$, let us first define the Dirichlet-to-Neumann operator

$$
\begin{aligned}
\Lambda_{R, \boldsymbol{h}}: \mathrm{H}^{s+1 / 2}\left(\partial \mathcal{B}_{R}\right) & \rightarrow \mathrm{H}^{s-1 / 2}\left(\partial \mathcal{B}_{R}\right) \\
\boldsymbol{\varphi} & \mapsto \frac{R(1+\nu)}{E} \sigma\left(\mathbf{v}_{\boldsymbol{h}}\right) \cdot \mathbf{n},
\end{aligned}
$$

where $\mathbf{v}_{\boldsymbol{h}} \in \mathrm{H}^{s+1}\left(\mathcal{B}_{R} \backslash \omega_{\boldsymbol{h}}\right)$ solves the boundary value problem (3.3) with $\omega=\omega_{\boldsymbol{h}}$. We are now in a position to state the result of this section.

Proposition 3.6. In the previously described geometric setting, and for all $s \geq 0$, there exists $C_{s}$ such that if $\|\boldsymbol{h}\|_{\mathrm{W}^{1, \infty}\left(\mathcal{B}_{R}\right)}<1$, then

$$
\left\|\Lambda_{R, \boldsymbol{h}}-\Lambda_{R}\right\|_{\mathcal{L}\left(\mathrm{H}^{s+1 / 2}\left(\partial \mathcal{B}_{R}\right), \mathrm{H}^{s-1 / 2}\left(\partial \mathcal{B}_{R}\right)\right)} \leq C_{s}\|\boldsymbol{h}\|_{\mathrm{W}^{1, \infty}\left(\mathcal{B}_{R}\right)} .
$$

Proof. The difference with the situation presented in [9] is that one deals with the elasticity system instead of the Laplace equation. Hence the transported weak formation for (3.3) is more complicated: one has to transport the symmetrized gradient instead of the usual gradient. Its reads

$$
e_{\boldsymbol{h}}(\mathbf{u})=\frac{1}{2}\left(D \mathbf{u} \cdot D \mathrm{~T}_{\boldsymbol{h}}^{-1}+\left(D \mathrm{~T}_{\boldsymbol{h}}^{-1}\right)^{\top} \cdot D \mathbf{u}^{\top}\right) .
$$

For example, the starting point is to write the perturbed bilinear form as

$$
a(\mathbf{v}, \boldsymbol{\varphi})=\int_{\mathcal{B}_{R} \backslash \mathcal{B}_{1}} e_{\boldsymbol{h}}(\mathbf{v}): H: e_{\boldsymbol{h}}(\boldsymbol{\varphi}) \operatorname{det} D \mathrm{~T}_{\boldsymbol{h}},
$$

with $\mathbf{v}=\mathbf{u} \circ \mathrm{T}_{h}$. Then, one adapts straightforwardly step by step the proof of Theorem 3.1 in $[9$. This is left to the reader. 
3.5. Proof of Theorem 1.1. Now we can complete the proof of Theorem 1.1, First notice that point (1) corresponding to the case when $\omega=\mathcal{B}_{1}$ is a rephrasing of points (3) and (4) of Proposition 3.4. We can anyway repeat the argument for point (4) in a slightly more direct formulation, which will be useful for the proof of point (2).

Proof of point (1) of Theorem 1.1 when $R \rightarrow \infty$ (Alternative). As seen when the general strategy was explained in Section 3.1, solvability of problem (1.4) is equivalent to solving equation (3.5) on $\partial \mathcal{B}_{R}$. We introduce the following operator:

$$
\mathcal{P}_{R}^{0} \boldsymbol{\varphi}=\frac{1}{2}\left[\begin{array}{cc}
-\frac{\nu}{2(1-\nu)} & 0 \\
0 & \frac{1-\nu}{1-2 \nu}
\end{array}\right] \partial_{\theta}^{2} \boldsymbol{\varphi}+\boldsymbol{\varphi}+\Lambda_{R}^{0}(\boldsymbol{\varphi}),
$$

and we work now with parameter $\gamma$ instead of parameter $\nu$. Then from point (1) of Proposition 3.4 and Remark 3.1. we know that for any $\gamma \notin \mathcal{S}$, fixed from now on, the problem

$$
\mathcal{P}_{R}^{0} \boldsymbol{\varphi}=-\frac{R(1+\nu)}{E} \sigma\left(\mathbf{u}_{0}\right) \cdot \mathbf{n}
$$

is well posed with a unique solution $\varphi \in \mathrm{H}^{5 / 2}\left(\partial \mathcal{B}_{R}\right) \subset \mathrm{H}^{2}\left(\partial \mathcal{B}_{R}\right)$. This is due to the fact that $\mathcal{P}_{R}^{0}$ is elliptic of order 2 and invertible from $\mathrm{H}^{s+3 / 2}\left(\partial \mathcal{B}_{R}\right)$ to $\mathrm{H}^{s-1 / 2}\left(\partial \mathcal{B}_{R}\right)$ for all $s \in \mathbb{R}$, according to the Fourier mode decomposition given in Proposition 3.2 . Recall that ellipticity of $\mathcal{P}_{R}^{0}$ is a consequence of the fact that the Dirichlet-toNeumann map $\Lambda_{R}^{0}$ is of order 1 . Another consequence is that $\mathcal{P}_{R}^{0}$ has compact resolvent, and therefore a discrete spectrum as an unbounded operator in $L^{2}\left(\partial \mathcal{B}_{R}\right)$ (this partly explains the countability argument and the introduction of the countable set $\mathcal{R}_{\gamma}$ ). The invertibility of $\mathcal{P}_{R}^{0}$ is then equivalent to saying that 0 is not in the spectrum of $\mathcal{P}_{R}^{0}$. If we introduce the so-called resolvent at 0 defined by $\left(\mathcal{P}_{R}^{0}\right)^{-1}: \mathrm{L}^{2}\left(\partial \mathcal{B}_{R}\right) \longrightarrow \mathrm{L}^{2}\left(\partial \mathcal{B}_{R}\right)$, we can write

$$
\boldsymbol{\varphi}=\left(\mathcal{P}_{R}^{0}\right)^{-1}\left(-\frac{R(1+\nu)}{E} \sigma\left(\mathbf{u}_{0}\right) \cdot \mathbf{n}\right)
$$

recalling that $-\frac{R(1+\nu)}{E} \sigma\left(\mathbf{u}_{0}\right) \cdot \mathbf{n} \in \mathrm{H}^{1 / 2}\left(\partial \mathcal{B}_{R}\right)$. Since the spectrum is discrete, and 0 is not in the spectrum, there exists $\varepsilon_{\gamma}$ such that for all bounded operators $\mathcal{Q}_{R}$ : $\mathrm{H}^{2}\left(\partial \mathcal{B}_{R}\right) \longrightarrow \mathrm{L}^{2}\left(\partial \mathcal{B}_{R}\right)$ with norm strictly less than $\varepsilon_{\gamma}$, there also exists a unique solution to the problem

$$
\left(\mathcal{P}_{R}^{0}+\mathcal{Q}_{R}\right) \boldsymbol{\varphi}=-\frac{R(1+\nu)}{E} \sigma\left(\mathbf{u}_{0}\right) \cdot \mathbf{n} .
$$

For this it is sufficient to take $\varepsilon_{\gamma}=\left\|\mathcal{P}_{R}^{0}\right\|_{\mathcal{L}\left(\mathrm{H}^{2}\left(\partial \mathcal{B}_{R}\right), \mathrm{L}^{2}\left(\partial \mathcal{B}_{R}\right)\right)}^{-1}$, and the corresponding inverse is then given by

$$
\left(\mathcal{P}_{R}^{0}+\mathcal{Q}_{R}\right)^{-1}=\left(\mathcal{P}_{R}^{0}\right)^{-1}\left(\operatorname{Id}+\mathcal{Q}_{R}\left(\mathcal{P}_{R}^{0}\right)^{-1}\right)^{-1},
$$

where the first inverse is well defined using the Neumann series and $\left\|\mathcal{Q}_{R}\left(\mathcal{P}_{R}^{0}\right)^{-1}\right\|<$ 1.

We apply this strategy to $\mathcal{Q}_{R}=\mathcal{R}=\Lambda_{R}-\Lambda_{R}^{0}$ for which we showed in Proposition 3.3 that

$$
\|\mathcal{R}\|_{\mathcal{L}\left(\mathrm{L}^{2}\left(\partial \mathcal{B}_{R}\right), \mathrm{L}^{2}\left(\partial \mathcal{B}_{R}\right)\right)} \leq C_{0}(\gamma) R^{-2}
$$

(recall that the norm of operator $\mathcal{P}_{R}^{0}$ is independent of $R$ from its expression in Fourier modes). We therefore get that for $R$ such that $C_{0}(\gamma) R^{-2}<\varepsilon_{\gamma}$, problem (3.5) is well posed, with a solution $\varphi \in \mathrm{H}^{5 / 2}\left(\partial \mathcal{B}_{R}\right) \subset \mathrm{H}^{3 / 2}\left(\partial \mathcal{B}_{R}\right)$. This concludes 
the alternative proof of part (1) of Theorem 1.1 concerning the existence of $R_{\gamma}$, called $R_{\nu}$ in the statement.

Proof of part (2) of Theorem 1.1. We apply exactly the same argument in the case when $\omega=\omega_{\boldsymbol{h}}$ is close to the unit ball in the sense of Subsection 3.4. In that case, we want to solve on $\partial \mathcal{B}_{R}$ :

$$
\frac{1}{2}\left[\begin{array}{cc}
-\frac{\nu}{2(1-\nu)} & 0 \\
0 & \frac{1-\nu}{1-2 \nu}
\end{array}\right] \partial_{\theta}^{2} \boldsymbol{\varphi}+\boldsymbol{\varphi}+\Lambda_{R, \boldsymbol{h}}(\boldsymbol{\varphi})=-\frac{R(1+\nu)}{E} \sigma\left(\mathbf{u}_{0}\right) \cdot \mathbf{n} .
$$

This can be rewritten as

$$
\left(\mathcal{P}_{R}^{0}+\mathcal{Q}_{R}\right) \boldsymbol{\varphi}=-\frac{R(1+\nu)}{E} \sigma\left(\mathbf{u}_{0}\right) \cdot \mathbf{n}, \quad \text { with } \mathcal{Q}_{R}=\left(\Lambda_{R}-\Lambda_{R}^{0}\right)+\left(\Lambda_{R, h}-\Lambda_{R}\right) .
$$

We use Propositions 3.3 and 3.6 to get

$$
\left\|\mathcal{Q}_{R}\right\|_{\mathcal{L}\left(\mathrm{H}^{1}\left(\partial \mathcal{B}_{R}\right), L^{2}\left(\partial \mathcal{B}_{R}\right)\right)} \leq C_{1}(\gamma) R^{-2}+C_{1 / 2}\|\boldsymbol{h}\|_{\mathrm{W}^{1, \infty}\left(\mathcal{B}_{R}\right)},
$$

where $C_{1}(\gamma)$ and $C_{1 / 2}$ are defined in Propositions 3.3 and 3.6 respectively. Choosing $R_{\gamma}$ such that $C_{1}(\gamma) R_{\gamma}^{-2}<\varepsilon_{\gamma} / 2$ and $C_{1 / 2}\|\boldsymbol{h}\|_{\mathrm{W}^{1, \infty}\left(\mathcal{B}_{R}\right)}<\varepsilon_{\gamma} / 2$ give the result of part (2) of Theorem 1.1, with $R_{\nu}=R_{\gamma}$ and $\varepsilon_{\nu}=C_{1 / 2}^{-1} \varepsilon_{\gamma} / 2$ there.

\section{Proof of Proposition 3.2}

In this section, we give the proof of Proposition 3.2 This will be done in several steps. The first one is to analyze equation $L \mathbf{v}=0$ in Fourier modes and seek solutions which will appear to have a special form. The second one is to make explicit mode by mode the expression of

$$
\frac{r(1+\nu)}{E} \sigma(\mathbf{v}) \cdot \mathbf{n} \quad \text { on } \partial \mathcal{B}_{r} .
$$

The third one is to use boundary conditions in (3.3) for $r=1$ and $r=R$ to get the expression of the Dirichlet-to-Neumann map mode by mode (this will be split into cases $n \geq 2, n=1$ and $n=0)$.

4.1. First step: solutions of $L \mathbf{v}=0$ in Fourier modes. We shall look for solutions $\mathbf{v}$ in polar coordinates in the form of a Fourier series:

$$
\mathbf{v}=\left[\begin{array}{l}
v^{1} \\
v^{2}
\end{array}\right]=\left[\begin{array}{l}
e_{0}^{r}(r) \\
e_{0}^{\theta}(r)
\end{array}\right]+\sum_{n \geq 1}\left[\begin{array}{l}
c_{n}^{r}(r) \\
c_{n}^{\theta}(r)
\end{array}\right] \cos n \theta+\sum_{n \geq 1}\left[\begin{array}{l}
d_{n}^{r}(r) \\
d_{n}^{\theta}(r)
\end{array}\right] \sin n \theta .
$$

We first stick to the case when $n \geq 1$. It will appear later that we have to separate the study between the cases $n=1$ and $n \geq 2$. Using the expression (2.1) of the elasticity operator in polar coordinates, the coefficients $\left(c_{n}^{r}, c_{n}^{\theta}, d_{n}^{r}, d_{n}^{\theta}\right)$ satisfy

$$
\begin{aligned}
& (\lambda+2 \mu)\left(c_{n}^{r \prime \prime}+\frac{1}{r} c_{n}^{r^{\prime}}-\frac{1}{r^{2}} c_{n}^{r}\right)-\frac{\mu n^{2}}{r^{2}} c_{n}^{r}-\frac{\lambda+3 \mu}{r^{2}} n d_{n}^{\theta}+\frac{\lambda+\mu}{r} n d_{n}^{\theta^{\prime}}=0, \\
& (\lambda+2 \mu)\left(d_{n}^{r \prime \prime}+\frac{1}{r} d_{n}^{r^{\prime}}-\frac{1}{r^{2}} d_{n}^{r}\right)-\frac{\mu n^{2}}{r^{2}} d_{n}^{r}+\frac{\lambda+3 \mu}{r^{2}} n c_{n}^{\theta}-\frac{\lambda+\mu}{r} n c_{n}^{\theta^{\prime}}=0, \\
& \mu\left(c_{n}^{\theta^{\prime \prime}}+\frac{1}{r} c_{n}^{\theta^{\prime}}-\frac{1}{r^{2}} c_{n}^{\theta}\right)-\frac{\lambda+2 \mu}{r^{2}} n^{2} c_{n}^{\theta}+\frac{\lambda+\mu}{r} n d_{n}^{r \prime}+\frac{\lambda+3 \mu}{r^{2}} n d_{n}^{r}=0, \\
& \mu\left(d_{n}^{\theta^{\prime \prime}}+\frac{1}{r} d_{n}^{\theta^{\prime}}-\frac{1}{r^{2}} d_{n}^{\theta}\right)-\frac{\lambda+2 \mu}{r^{2}} n^{2} d_{n}^{\theta}-\frac{\lambda+\mu}{r} n c_{n}^{r^{\prime}}-\frac{\lambda+3 \mu}{r^{2}} n c_{n}^{r}=0
\end{aligned}
$$


This is a system of four differential equations which are coupled two by two. We introduce

$$
\gamma=\frac{\mu}{\lambda+2 \mu}=\frac{1-2 \nu}{2(1-\nu)} \in\left(0, \frac{3}{4}\right) .
$$

Then relations (4.2)- (4.5) rewrite as

$$
\begin{aligned}
& \left\{\begin{array}{l}
\left(c_{n}^{r \prime \prime}+\frac{1}{r} c_{n}^{r \prime}-\frac{1}{r^{2}} c_{n}^{r}-\frac{n}{r^{2}} d_{n}^{\theta}+\frac{n}{r} d_{n}^{\theta^{\prime}}\right)-\frac{\gamma n}{r}\left(\frac{n}{r} c_{n}^{r}+\frac{1}{r} d_{n}^{\theta}+d_{n}^{\theta^{\prime}}\right)=0 \\
\gamma\left(d_{n}^{\theta^{\prime \prime}}+\frac{1}{r} d_{n}^{\theta^{\prime}}-\frac{1}{r^{2}} d_{n}^{\theta}-\frac{n}{r^{2}} c_{n}^{r}+\frac{n}{r} c_{n}^{r \prime}\right)-\frac{n}{r}\left(\frac{n}{r} d_{n}^{\theta}+\frac{1}{r} c_{n}^{r}+c_{n}^{r \prime}\right)=0
\end{array}\right. \\
& \left\{\begin{array}{l}
\left(d_{n}^{r \prime \prime}+\frac{1}{r} d_{n}^{r^{\prime}}-\frac{1}{r^{2}} d_{n}^{r}+\frac{n}{r^{2}} c_{n}^{\theta}-\frac{n}{r} c_{n}^{\theta^{\prime}}\right)-\frac{\gamma n}{r}\left(\frac{n}{r} d_{n}^{r}-\frac{1}{r} c_{n}^{\theta}-c_{n}^{\theta^{\prime}}\right)=0 \\
\gamma\left(c_{n}^{\theta^{\prime \prime}}+\frac{1}{r} c_{n}^{\theta^{\prime}}-\frac{1}{r^{2}} c_{n}^{\theta}+\frac{n}{r^{2}} d_{n}^{r}-\frac{n}{r} d_{n}^{r^{\prime}}\right)-\frac{n}{r}\left(\frac{n}{r} c_{n}^{\theta}-\frac{1}{r} d_{n}^{r}-d_{n}^{r \prime}\right)=0
\end{array}\right.
\end{aligned}
$$

Mimicking the form of the solutions in the Fourier series for the Laplace problem, we look for solutions of the form

$$
\begin{aligned}
& c_{n}^{r}(r)=\beta_{r} r^{\alpha} \quad \text { and } \quad d_{n}^{\theta}(r)=\beta_{\theta} r^{\alpha}, \\
& d_{n}^{r}(r)=\tilde{\beta}_{r} r^{\tilde{\alpha}} \quad \text { and } \quad c_{n}^{\theta}(r)=\tilde{\beta}_{\theta} r^{\tilde{\alpha}} \text {, }
\end{aligned}
$$

where the parameters $\alpha, \beta$ and $\tilde{\alpha}, \tilde{\beta}$ have to be determined. Substituting these expressions into (4.7)-(4.8), we obtain

$$
\begin{gathered}
\begin{cases}\left(\left(\alpha^{2}-1\right) \beta_{r}+n(\alpha-1) \beta_{\theta}\right)-\gamma n\left(n \beta_{r}+(\alpha+1) \beta_{\theta}\right) & =0, \\
\gamma\left(\left(\alpha^{2}-1\right) \beta_{\theta}+n(\alpha-1) \beta_{r}\right)-n\left(n \beta_{\theta}+(\alpha+1) \beta_{r}\right) & =0,\end{cases} \\
\begin{cases}\left(\left(\tilde{\alpha}^{2}-1\right) \tilde{\beta}_{r}-n(\tilde{\alpha}-1) \tilde{\beta}_{\theta}\right)-\gamma n\left(n \tilde{\beta}_{r}-(\tilde{\alpha}+1) \tilde{\beta}_{\theta}\right) & =0, \\
\gamma\left(\left(\tilde{\alpha}^{2}-1\right) \tilde{\beta}_{\theta}-n(\tilde{\alpha}-1) \tilde{\beta}_{r}\right)-n\left(n \tilde{\beta}_{\theta}-(\tilde{\alpha}+1) \tilde{\beta}_{r}\right) & =0 .\end{cases}
\end{gathered}
$$

In a matrix form, these equations read

$$
M(\alpha)\left[\begin{array}{l}
\beta_{r} \\
\beta_{\theta}
\end{array}\right]=\left[\begin{array}{l}
0 \\
0
\end{array}\right] \quad \text { and } \quad \widetilde{M}(\tilde{\alpha})\left[\begin{array}{c}
\tilde{\beta}_{r} \\
\tilde{\beta}_{\theta}
\end{array}\right]=\left[\begin{array}{l}
0 \\
0
\end{array}\right],
$$

with

$$
M(\alpha)=\left[\begin{array}{cc}
\alpha^{2}-1-\gamma n^{2} & n(\alpha-1-\gamma(\alpha+1)) \\
n(\gamma(\alpha-1)-(\alpha+1)) & \gamma\left(\alpha^{2}-1\right)-n^{2}
\end{array}\right]
$$

and

$$
\widetilde{M}(\alpha)=\left[\begin{array}{cc}
\alpha^{2}-1-\gamma n^{2} & -n(\alpha-1-\gamma(\alpha+1)) \\
-n(\gamma(\alpha-1)-(\alpha+1)) & \gamma\left(\alpha^{2}-1\right)-n^{2}
\end{array}\right]
$$

Thus, the determinants of the later two matrices $M(\alpha)$ and $\widetilde{M}(\alpha)$ involve the same biquadratic expression in $\alpha$ and are given by

$$
\operatorname{det} M(\alpha)=\operatorname{det} \widetilde{M}(\alpha)=\gamma\left(\alpha^{4}-2 \alpha^{2}\left(1+n^{2}\right)+\left(n^{2}-1\right)^{2}\right) .
$$

These determinants cancel for

$$
\alpha_{n}^{ \pm \pm}= \pm n \pm 1 .
$$

At this point we notice that we have four roots when $n \geq 2$ and only three in the case when $n=1$ for which $\alpha_{1}^{-+}=\alpha_{1}^{+-}=0$. We study the two cases separately. 
4.1.1. Case $n \geq 2$. Consider the four roots $\alpha_{n}^{ \pm \pm}$. For each of them and modulo a multiplicative constant, the coordinates $\beta_{r, n}^{ \pm \pm}, \beta_{\theta, n}^{ \pm \pm}, \tilde{\beta}_{r, n}^{ \pm \pm}, \tilde{\beta}_{\theta, n}^{ \pm \pm}$of vectors satisfying (4.13) are defined by

$$
\begin{array}{ll}
\beta_{\theta, n}^{ \pm \pm}=\frac{1}{\gamma-1}\left(\gamma-\frac{\left(\alpha_{n}^{ \pm \pm}\right)^{2}-1}{n^{2}}\right), & \beta_{r, n}^{ \pm \pm}=\frac{\alpha_{n}^{ \pm \pm}-1-\gamma\left(\alpha_{n}^{ \pm \pm}+1\right)}{n(\gamma-1)}, \\
\tilde{\beta}_{\theta, n}^{ \pm \pm}=\beta_{\theta, n}^{ \pm \pm}, & \tilde{\beta}_{r, n}^{ \pm \pm}=-\beta_{r, n}^{ \pm \pm} .
\end{array}
$$

After simplification, we have

$$
\beta_{\theta, n}^{ \pm \pm}=1-\frac{ \pm \pm 2}{n(\gamma-1)}, \quad \beta_{r, n}^{ \pm \pm}=-\frac{\alpha_{n}^{ \pm \pm}+1}{n}-\frac{2}{n(\gamma-1)} .
$$

For each choice $\alpha_{n}^{ \pm \pm}= \pm n \pm 1$, we choose explicitly the coefficients $\beta_{\theta, n}^{ \pm \pm}, \beta_{r, n}^{ \pm \pm}$by (4.15)

$$
\left\{\begin{array}{lll}
\alpha_{n}^{++}=n+1, & \beta_{\theta, n}^{++}=1-\frac{2}{n(\gamma-1)}, & \beta_{r, n}^{++}=-1-\frac{2 \gamma}{n(\gamma-1)}, \\
\alpha_{n}^{+-}=n-1, & \beta_{\theta, n}^{+-}=1, & \beta_{r, n}^{+-}=-1, \\
\alpha_{n}^{-+}=-n+1, & \beta_{\theta, n}^{-+}=1+\frac{2}{n(\gamma-1)}, & \beta_{r, n}^{-+}=1-\frac{2 \gamma}{n(\gamma-1)}, \\
\alpha_{n}^{--}=-n-1, & \beta_{\theta, n}^{--}=1, & \beta_{r, n}^{--}=1 .
\end{array}\right.
$$

Then the functions $c_{n}^{r}, d_{n}^{\theta}, d_{n}^{r}, c_{n}^{\theta}$ take the form

$$
\begin{aligned}
& c_{n}^{r}(r)=\beta_{r, n}^{--} A_{n}^{--} r^{-n-1}+\beta_{r, n}^{-+} A_{n}^{-+} r^{-n+1}+\beta_{r, n}^{+-} A_{n}^{+-} r^{n-1}+\beta_{r, n}^{++} A_{n}^{++} r^{n+1}, \\
& d_{n}^{\theta}(r)=\beta_{\theta, n}^{--} A_{n}^{--} r^{-n-1}+\beta_{\theta, n}^{-+} A_{n}^{-+} r^{-n+1}+\beta_{\theta, n}^{+-} A_{n}^{+-} r^{n-1}+\beta_{\theta, n}^{++} A_{n}^{++} r^{n+1}, \\
& d_{n}^{r}(r)=-\beta_{r, n}^{--} B_{n}^{--} r^{-n-1}-\beta_{r, n}^{-+} B_{n}^{-+} r^{-n+1}-\beta_{r, n}^{+-} B_{n}^{+-} r^{n-1}-\beta_{r, n}^{++} B_{n}^{++} r^{n+1}, \\
& c_{n}^{\theta}(r)=\beta_{\theta, n}^{--} B_{n}^{--} r^{-n-1}+\beta_{\theta, n}^{-+} B_{n}^{-+} r^{-n+1}+\beta_{\theta, n}^{+-} B_{n}^{+-} r^{n-1}+\beta_{\theta, n}^{++} B_{n}^{++} r^{n+1} .
\end{aligned}
$$

After simplification using (4.15), we obtain

$$
\begin{aligned}
& c_{n}^{r}(r)=A_{n}^{--} r^{-n-1}+\beta_{r, n}^{-+} A_{n}^{-+} r^{-n+1}-A_{n}^{+-} r^{n-1}+\beta_{r, n}^{++} A_{n}^{++} r^{n+1} \\
& d_{n}^{\theta}(r)=A_{n}^{--} r^{-n-1}+\beta_{\theta, n}^{-+} A_{n}^{-+} r^{-n+1}+A_{n}^{+-} r^{n-1}+\beta_{\theta, n}^{++} A_{n}^{++} r^{n+1} \\
& d_{n}^{r}(r)=-B_{n}^{--} r^{-n-1}-\beta_{r, n}^{-+} B_{n}^{-+} r^{-n+1}+B_{n}^{+-} r^{n-1}-\beta_{r, n}^{++} B_{n}^{++} r^{n+1} \\
& c_{n}^{\theta}(r)=B_{n}^{--} r^{-n-1}+\beta_{\theta, n}^{-+} B_{n}^{-+} r^{-n+1}+B_{n}^{+-} r^{n-1}+\beta_{\theta, n}^{++} B_{n}^{++} r^{n+1}
\end{aligned}
$$

For each $n \geq 2$, systems (4.7) and (4.8) are systems of two linear differential equations of second order, therefore with a vector space of solutions of dimension four. On the other hand the solutions described in (4.16)-(4.17), respectively (4.18)(4.19), each span a vector space of dimension four as the four real numbers $A_{n}^{ \pm \pm}$ vary. We have therefore found all the solutions of respectively (4.7) and (4.8) for $n \geq 2$.

4.1.2. Case $n=1$. In this subsection, we keep the subscript $n$ although $n=1$. In this case, we have three roots $\alpha_{n}^{++}=2, \alpha_{n}^{00}=0$ and $\alpha_{n}^{--}=-2$. Associated to each of them we can choose coordinates of eigenvectors in the following way:

$$
\left\{\begin{array}{rlrl}
\alpha_{n}^{++} & =2, & \beta_{\theta, n}^{++}=1-\frac{2}{\gamma-1}, & \beta_{r, n}^{++}=-1-\frac{2 \gamma}{\gamma-1} \\
\alpha_{n}^{00}=0, & \beta_{\theta, n}^{00}=1, & \beta_{r, n}^{00}=-1, \\
\alpha_{n}^{--}=-2, & \beta_{\theta, n}^{--}=1, & \beta_{r, n}^{--}=1 .
\end{array}\right.
$$


Then (for $n=1$ ) the functions $c_{n}^{r}, d_{n}^{\theta}, d_{n}^{r}, c_{n}^{\theta}$ take the form

$$
\begin{aligned}
& c_{n}^{r}(r)=\beta_{r, n}^{--} A_{n}^{--} r^{-n-1}+\beta_{r, n}^{00} A_{n}^{00} r^{n-1}+\beta_{r, n}^{++} A_{n}^{++} r^{n+1}, \\
& d_{n}^{\theta}(r)=\beta_{\theta, n}^{--} A_{n}^{--} r^{-n-1}+\beta_{\theta, n}^{00} A_{n}^{00} r^{n-1}+\beta_{\theta, n}^{++} A_{n}^{++} r^{n+1}, \\
& d_{n}^{r}(r)=-\beta_{r, n}^{--} B_{n}^{--} r^{-n-1}-\beta_{r, n}^{00} B_{n}^{00} r^{n-1}-\beta_{r, n}^{++} B_{n}^{++} r^{n+1}, \\
& c_{n}^{\theta}(r)=\beta_{\theta, n}^{--} B_{n}^{--} r^{-n-1}+\beta_{\theta, n}^{00} B_{n}^{00} r^{n-1}+\beta_{\theta, n}^{++} B_{n}^{++} r^{n+1} .
\end{aligned}
$$

After simplification using (4.20), we obtain

$$
\begin{aligned}
& c_{n}^{r}(r)=A_{n}^{--} r^{-2}-A_{n}^{00}+\beta_{r, n}^{++} A_{n}^{++} r^{2}, \\
& d_{n}^{\theta}(r)=A_{n}^{--} r^{-2}+A_{n}^{00}+\beta_{\theta, n}^{++} A_{n}^{++} r^{2}, \\
& d_{n}^{r}(r)=-B_{n}^{--} r^{-2}+B_{n}^{00}-\beta_{r, n}^{++} B_{n}^{++} r^{2}, \\
& c_{n}^{\theta}(r)=B_{n}^{--} r^{-2}+B_{n}^{00}+\beta_{\theta, n}^{++} B_{n}^{++} r^{2} .
\end{aligned}
$$

Let us first prove that we have found the full space of solutions of (4.7) and (4.8) for $n=1$.

Lemma 4.1. The space of solutions of systems (4.7) and (4.8) are a three-dimensional space and any solution is given by (4.21)-(4.24).

Proof. We prove this result for system (4.7). The proof is essentially similar for system (4.8).

We use the change in variables $r=e^{x}$ and define

$$
c_{n}^{r}(r)=f(\ln r), \quad d_{n}^{\theta}(r)=g(\ln r) .
$$

System (4.7) can be rewritten after simplification:

$$
\left\{\begin{array}{l}
\left(f^{\prime \prime}-f-g+g^{\prime}\right)-\gamma\left(f+g+g^{\prime}\right)=0 \\
\gamma\left(g^{\prime \prime}-g-f+f^{\prime}\right)-\left(g+f+f^{\prime}\right)=0 .
\end{array}\right.
$$

Denoting by $\mathbf{U}=\left(f, g, f^{\prime}, g^{\prime}\right)^{\top}$, we write (4.25) on the form $\mathbf{U}^{\prime}=M \mathbf{U}$ with

$$
M=\left[\begin{array}{cccc}
0 & 0 & 1 & 0 \\
0 & 0 & 0 & 1 \\
1+\gamma & 1+\gamma & 0 & \gamma-1 \\
1+\frac{1}{\gamma} & 1+\frac{1}{\gamma} & \frac{1}{\gamma}-1 & 0
\end{array}\right]
$$

We have $\operatorname{det}\left(M-X \mathrm{Id}_{4}\right)=X^{2}(X-2)(X+2)$. We notice that we again find the solutions $e^{2 x}=r^{2}, e^{-2 x}=r^{-2}$, and the constant functions. Computing a Jordan decomposition for $M$, we have

$$
M=P T P^{-1},
$$

with

$$
T=\left[\begin{array}{cccc}
2 & 0 & 0 & 0 \\
0 & -2 & 0 & 0 \\
0 & 0 & 0 & 1 \\
0 & 0 & 0 & 0
\end{array}\right] \quad \text { and } \quad P=\left[\begin{array}{cccc}
3 \gamma-1 & 1 & 1 & 0 \\
3-\gamma & 1 & -1 & 0 \\
2(3 \gamma-1) & -2 & 0 & 1 \\
2(3-\gamma) & -2 & 0 & -1
\end{array}\right]
$$


The vector $\mathbf{V}=P^{-1} \mathbf{U}$ satisfies the equation $\mathbf{V}^{\prime}=T \mathbf{V}$ whose general solution is given by

We infer

$$
\mathbf{V}(x)=\left[\begin{array}{c}
a e^{2 x} \\
b e^{-2 x} \\
c \\
c x+d
\end{array}\right], \quad \text { with } \quad a, b, c, d \in \mathbb{R}^{4} .
$$

$$
\mathbf{U}(x)=P \mathbf{V}(x)=\left[\begin{array}{c}
a(3 \gamma-1) e^{2 x}+b e^{-2 x}+c \\
a(3-\gamma) e^{2 x}+b e^{-2 x}-c \\
2 a(3 \gamma-1) e^{2 x}-2 b e^{-2 x}+c x+d \\
2 a(3-\gamma) e^{2 x}-2 b e^{-2 x}-c x-d
\end{array}\right]
$$

Coming back to the initial $r$-variable, the first two relations give the general expression for $c_{n}^{r}$ and $d_{n}^{\theta}$ :

$$
\left[\begin{array}{l}
c_{n}^{r}(r) \\
d_{n}^{\theta}(r)
\end{array}\right]=\left[\begin{array}{c}
a(3 \gamma-1) r^{2}+b r^{-2}+c \\
a(3-\gamma) r^{2}+b r^{-2}-c
\end{array}\right]=\left[\begin{array}{c}
a(1-\gamma) \beta_{r, n}^{++} r^{2}+b r^{-2}+c \\
a(1-\gamma) \beta_{\theta, n}^{++} r^{2}+b r^{-2}-c
\end{array}\right],
$$

since $\beta_{r, n}^{++}=\frac{1-3 \gamma}{\gamma-1}$ and $\beta_{\theta, n}^{++}=\frac{\gamma-3}{\gamma-1}$. This finishes the proof of the lemma.

4.1.3. Case $n=0$. System (2.1) then simply reads

$$
\begin{aligned}
(\lambda+2 \mu)\left(e_{0}^{r \prime \prime}+\frac{1}{r} e_{0}^{r \prime}-\frac{1}{r^{2}} e_{0}^{r}\right) & =0, \\
\mu\left(e_{0}^{\theta^{\prime \prime}}+\frac{1}{r} e_{0}^{\theta^{\prime}}-\frac{1}{r^{2}} e_{0}^{\theta}\right) & =0 .
\end{aligned}
$$

This is a decoupled system of two second order equations, for which the solutions are easily shown to be

$$
\begin{aligned}
& e_{0}^{r}(r)=A_{0}^{--} r^{-1}+A_{0}^{++} r, \\
& e_{0}^{\theta}(r)=B_{0}^{--} r^{-1}+B_{0}^{++} r .
\end{aligned}
$$

Thus we have found the full vector space of solutions for $n=0$.

4.2. Expression of the Neumann operator. In this section, we give the expression of the Neumann operator

$$
\frac{r(1+\nu)}{E} \sigma(\mathbf{v}) \cdot \mathbf{n}(r)
$$

first when $\mathbf{v}$ has the form (4.1), and then in the special case when $\mathbf{v}$ is a solution of $L \mathbf{v}$ and satisfies (4.16)-(4.19), (4.21)-(4.24) and (4.28)-(4.29). First using (2.2) and (4.1), we can write

$$
\begin{aligned}
\sigma(\mathbf{v}) \cdot \mathbf{n}(r)= & {\left[\begin{array}{c}
(\lambda+2 \mu) e_{0}^{r \prime}(r)+\frac{\lambda}{r} e_{0}^{r}(r) \\
\mu\left(e_{0}^{\theta^{\prime}}(r)-\frac{1}{r} e_{0}^{\theta}(r)\right)
\end{array}\right] } \\
& +\sum_{n \geq 1}\left[\begin{array}{c}
(\lambda+2 \mu) c_{n}^{r \prime}(r)+\frac{\lambda}{r} c_{n}^{r}(r)+\frac{\lambda}{r} n d_{n}^{\theta}(r) \\
\mu\left(\frac{n}{r} d_{n}^{r}(r)-\frac{1}{r} c_{n}^{\theta}(r)+c_{n}^{\theta^{\prime}}(r)\right)
\end{array}\right] \cos n \theta \\
& +\sum_{n \geq 1}\left[\begin{array}{c}
(\lambda+2 \mu) d_{n}^{r \prime}(r)+\frac{\lambda}{r} d_{n}^{r}(r)-\frac{\lambda}{r} n c_{n}^{\theta}(r) \\
\mu\left(-\frac{n}{r} c_{n}^{r}(r)-\frac{1}{r} d_{n}^{\theta}(r)+d_{n}^{\theta^{\prime}}(r)\right)
\end{array}\right] \sin n \theta .
\end{aligned}
$$


Using definition (4.6) of $\gamma$, we have $\frac{\lambda}{\lambda+2 \mu}=1-2 \gamma$ and we deduce

$$
\begin{aligned}
& r \sigma(\mathbf{v}) \cdot \mathbf{n}(r)= {\left[\begin{array}{cc}
\lambda+2 \mu & 0 \\
0 & \mu
\end{array}\right]\left(\left[\begin{array}{c}
e_{0}^{r \prime}(r) r+(1-2 \gamma) e_{0}^{r}(r) \\
e_{0}^{\theta^{\prime}}(r) r-e_{0}^{\theta}(r)
\end{array}\right]\right.} \\
&+\sum_{n \geq 1}\left[\begin{array}{c}
c_{n}^{r^{\prime}}(r) r+(1-2 \gamma) c_{n}^{r}(r)+n(1-2 \gamma) d_{n}^{\theta}(r) \\
n d_{n}^{r}(r)-c_{n}^{\theta}(r)+c_{n}^{\theta^{\prime}}(r) r
\end{array}\right] \cos n \theta \\
&\left.+\sum_{n \geq 1}\left[\begin{array}{c}
d_{n}^{r^{\prime}}(r) r+(1-2 \gamma) d_{n}^{r}(r)-n(1-2 \gamma) c_{n}^{\theta}(r) \\
-n c_{n}^{r}(r)-d_{n}^{\theta}(r)+d_{n}^{\theta^{\prime}}(r) r
\end{array}\right] \sin n \theta\right)
\end{aligned}
$$

Using (2.9), we also get that

$$
\frac{1+\nu}{E} \mu=\frac{1}{2} \quad \text { and } \quad \frac{1+\nu}{E}(\lambda+2 \mu)=\frac{1}{2 \gamma},
$$

and therefore

$$
\begin{aligned}
& \frac{1+\nu}{E} r \sigma(\mathbf{v}) \cdot \mathbf{n}(r)= {\left[\begin{array}{cc}
\frac{1}{2 \gamma} & 0 \\
0 & \frac{1}{2}
\end{array}\right]\left(\left[\begin{array}{c}
e_{0}^{r \prime}(r) r+(1-2 \gamma) e_{0}^{r}(r) \\
e_{0}^{\theta^{\prime}}(r) r-e_{0}^{\theta}(r)
\end{array}\right]\right.} \\
&+\sum_{n \geq 1}\left[\begin{array}{c}
c_{n}^{r \prime}(r) r+(1-2 \gamma) c_{n}^{r}(r)+n(1-2 \gamma) d_{n}^{\theta}(r) \\
n d_{n}^{r}(r)-c_{n}^{\theta}(r)+c_{n}^{\theta^{\prime}}(r) r
\end{array}\right] \cos n \theta \\
&+\sum_{n \geq 1}\left[\begin{array}{c}
\left.\left.d_{n}^{r \prime}(r) r+(1-2 \gamma) d_{n}^{r}(r)-n(1-2 \gamma) c_{n}^{\theta}(r)\right] \sin n \theta\right) . \\
-n c_{n}^{r}(r)-d_{n}^{\theta}(r)+d_{n}^{\theta^{\prime}}(r) r
\end{array}\right]
\end{aligned}
$$

For convenience we shall now write

$$
\begin{aligned}
& \forall n \geq 1, \quad\left\{\begin{array}{l}
c_{1}(n, r)=c_{n}^{r \prime}(r) r+(1-2 \gamma) c_{n}^{r}(r)+n(1-2 \gamma) d_{n}^{\theta}(r), \\
s_{2}(n, r)=-n c_{n}^{r}(r)-d_{n}^{\theta}(r)+d_{n}^{\theta^{\prime}}(r) r,
\end{array}\right. \\
& \forall n \geq 1, \quad\left\{\begin{array}{l}
s_{1}(n, r)=d_{n}^{r \prime}(r) r+(1-2 \gamma) d_{n}^{r}(r)-n(1-2 \gamma) c_{n}^{\theta}(r), \\
c_{2}(n, r)=n d_{n}^{r}(r)-c_{n}^{\theta}(r)+c_{n}^{\theta^{\prime}}(r) r,
\end{array}\right. \\
& c_{1}(r)=e_{0}^{r \prime}(r) r+(1-2 \gamma) e_{0}^{r}(r), \\
& c_{2}(r)=e_{0}^{\theta^{\prime}}(r) r-e_{0}^{\theta}(r),
\end{aligned}
$$

so that

$$
\begin{aligned}
& \frac{1+\nu}{E} r \sigma(\mathbf{v}) \cdot \mathbf{n}(r) \\
& \quad=\left[\begin{array}{cc}
\frac{1}{2 \gamma} & 0 \\
0 & \frac{1}{2}
\end{array}\right]\left(\left[\begin{array}{l}
c_{1}(r) \\
c_{2}(r)
\end{array}\right]+\sum_{n \geq 1}\left[\begin{array}{l}
c_{1}(n, r) \\
c_{2}(n, r)
\end{array}\right] \cos n \theta+\sum_{n \geq 1}\left[\begin{array}{l}
s_{1}(n, r) \\
s_{2}(n, r)
\end{array}\right] \sin n \theta\right) .
\end{aligned}
$$

In the following, we compute the coefficients $c_{1}(n, r), s_{2}(n, r), s_{1}(n, r), c_{2}(n, r)$, $c_{1}(r)$, and $c_{2}(r)$. Again we split the study depending on the cases $n \geq 2, n=1$ and $n=0$. 
4.2.1. Case $n \geq 2$. In that case, replacing $c_{n}^{r}$ and $d_{n}^{\theta}$ by their expression (4.16)(4.17) in (4.32)-4.33), we get

$$
\begin{aligned}
& c_{1}(n, r)=-2 \gamma(n+1) A_{n}^{--} r^{-n-1} \\
& +\left((-n+2-2 \gamma) \beta_{r, n}^{-+}+n(1-2 \gamma) \beta_{\theta, n}^{-+}\right) A_{n}^{-+} r^{-n+1} \\
& +2 \gamma(1-n) A_{n}^{+-} r^{n-1}+\left((n+2-2 \gamma) \beta_{r, n}^{++}+n(1-2 \gamma) \beta_{\theta, n}^{++}\right) A_{n}^{++} r^{n+1} \\
& =-2 \gamma(n+1) A_{n}^{--} r^{-n-1}+2 \gamma\left(\frac{2}{n}-1-n\right) A_{n}^{-+} r^{-n+1} \\
& +2 \gamma(1-n) A_{n}^{+-} r^{n-1}+2 \gamma\left(\frac{2}{n}+1-n\right) A_{n}^{++} r^{n+1}, \\
& s_{2}(n, r)=-2(n+1) A_{n}^{--} r^{-n-1}-n\left(\beta_{r, n}^{-+}+\beta_{\theta, n}^{-+}\right) A_{n}^{-+} r^{-n+1} \\
& +2(n-1) A_{n}^{+-} r^{n-1}-n\left(\beta_{r, n}^{++}-\beta_{\theta, n}^{++}\right) A_{n}^{++} r^{n+1} \\
& =-2(n+1) A_{n}^{--} r^{-n-1}+2(1-n) A_{n}^{-+} r^{-n+1} \\
& +2(n-1) A_{n}^{+-} r^{n-1}+2(n+1) A_{n}^{++} r^{n+1},
\end{aligned}
$$

and similarly with $c_{n}^{\theta}$ and $d_{n}^{r}$, we get

$$
\begin{gathered}
s_{1}(n, r)=-\left(-2 \gamma(n+1) B_{n}^{--} r^{-n-1}+2 \gamma\left(\frac{2}{n}-1-n\right) B_{n}^{-+} r^{-n+1}\right. \\
\left.+2 \gamma(1-n) B_{n}^{+-} r^{n-1}+2 \gamma\left(\frac{2}{n}+1-n\right) B_{n}^{++} r^{n+1}\right), \\
c_{2}(n, r)=-2(n+1) B_{n}^{--} r^{-n-1}-2(n-1) B_{n}^{-+} r^{-n+1} \\
-2(1-n) B_{n}^{+-} r^{n-1}+2(n+1) B_{n}^{++} r^{n+1} .
\end{gathered}
$$

4.2.2. Case $n=1$. A similar computation replacing $c_{n}^{r}$ and $d_{n}^{\theta}$ in (4.32)-(4.33) by their expression (4.21)-(4.24) gives in the case $n=1$,

$$
\begin{aligned}
& c_{1}(n, r)=-4 \gamma A_{n}^{--} r^{-2}+4 \gamma A_{n}^{++} r^{2}, \\
& s_{2}(n, r)=-4 A_{n}^{--} r^{-2}+4 A_{n}^{++} r^{2}, \\
& s_{1}(n, r)=4 \gamma B_{n}^{--} r^{-2}-4 \gamma B_{n}^{++} r^{2}, \\
& c_{2}(n, r)=-4 B_{n}^{--} r^{-2}+4 B_{n}^{++} r^{2} .
\end{aligned}
$$

Note that the constant terms $A_{n}^{00}$ and $B_{n}^{00}$ do not appear.

4.2.3. Case $n=0$. Using expressions (4.28) (4.29) in 4.34 4.35), we deduce

$$
\begin{aligned}
& c_{1}(r)=-2 \gamma A_{n}^{--} r^{-1}+2(1-\gamma) A_{n}^{++} r, \\
& c_{2}(r)=-2 B_{n}^{--} r^{-1} .
\end{aligned}
$$

4.3. DtN map and Ventcel conditions for modes $n \geq 2$. Now we are in a position to compute the expression of the Dirichlet-to-Neumann map for each mode $n \geq 2$ and prove the corresponding part of Proposition 3.2. The strategy is the following: We first get relations between the $A^{ \pm \pm}(n)$ thanks to the property in the inner disk of radius $r=1$. Then we get an expression of the component of $\varphi$ with respect to the predominant terms $A^{+ \pm}(n)$ thanks to the Dirichlet condition 
in (3.3), and we control the remainder terms (Lemma 4.2). Eventually we give the expression of $\Lambda_{R}(\varphi)$ with respect to $\varphi$ for mode $n$.

4.3.1. Neumann conditions on the inner circle. In this subsection we fix $n \geq 2$. In (3.3), we use the Neumann condition on the inner circle $\partial \omega$ of radius $r=1$ in order to get relations between the terms $A^{ \pm \pm}(n)$ (resp. $B^{ \pm \pm}(n)$ ). Neumann conditions also read

$$
\left.\frac{1+\nu}{E} r \sigma(\mathbf{v}) \cdot \mathbf{n}\right|_{r=1}=0 \text {. }
$$

This implies that for $r=1$ :

$$
c_{1}(n, r)=s_{2}(n, r)=0 \quad \text { and } \quad s_{1}(n, r)=c_{2}(n, r)=0 .
$$

Using (4.37)-(4.40), these equalities can be written in matrix form,

$$
M_{n}^{-}\left[\begin{array}{l}
A_{n}^{--} \\
A_{n}^{-+}
\end{array}\right]=M_{n}^{+}\left[\begin{array}{l}
A_{n}^{+-} \\
A_{n}^{++}
\end{array}\right] \quad \text { and } \quad M_{n}^{-}\left[\begin{array}{l}
B_{n}^{--} \\
B_{n}^{-+}
\end{array}\right]=M_{n}^{+}\left[\begin{array}{l}
B_{n}^{+-} \\
B_{n}^{++}
\end{array}\right],
$$

with the same matrices $M_{n}^{ \pm}$for the two linear systems defined by

$$
M_{n}^{-}=2\left[\begin{array}{cc}
-\gamma(n+1) & \gamma\left(\frac{2}{n}-1-n\right) \\
n+1 & n-1
\end{array}\right], \quad M_{n}^{+}=-2\left[\begin{array}{cc}
\gamma(1-n) & \gamma\left(\frac{2}{n}+1-n\right) \\
1-n & -(n+1)
\end{array}\right] .
$$

We have

$$
\operatorname{det} M_{n}^{-}=\operatorname{det} M_{n}^{+}=8 \gamma\left(n-\frac{1}{n}\right)
$$

We deduce

$$
\left(M_{n}^{-}\right)^{-1}=\frac{1}{4 \gamma\left(n-\frac{1}{n}\right)}\left[\begin{array}{cc}
n-1 & -\gamma\left(\frac{2}{n}-1-n\right) \\
-(n+1) & -\gamma(n+1)
\end{array}\right]=\varnothing(1) .
$$

Then, denoting $M_{n}=\left(M_{n}^{-}\right)^{-1} M_{n}^{+}$, we find

$$
M_{n}=\left[\begin{array}{cc}
n-1 & n \\
-n & -(n+1)
\end{array}\right] .
$$

With matrix $M_{n}$ given by (4.44), relations (4.43) read as

$$
\left[\begin{array}{l}
A_{n}^{--} \\
A_{n}^{-+}
\end{array}\right]=M_{n}\left[\begin{array}{l}
A_{n}^{+-} \\
A_{n}^{++}
\end{array}\right] \quad \text { and } \quad\left[\begin{array}{l}
B_{n}^{--} \\
B_{n}^{-+}
\end{array}\right]=M_{n}\left[\begin{array}{l}
B_{n}^{+-} \\
B_{n}^{++}
\end{array}\right] .
$$

Let us define

$$
M_{n}(R)=R^{-2 n}\left[\begin{array}{cc}
n-1 & n R^{-2} \\
-n R^{2} & -(n+1)
\end{array}\right]
$$

then

$$
\left[\begin{array}{l}
A_{n}^{--} R^{-n-1} \\
A_{n}^{-+} R^{-n+1}
\end{array}\right]=M_{n}(R)\left[\begin{array}{l}
A_{n}^{+-} R^{n-1} \\
A_{n}^{++} R^{n+1}
\end{array}\right], \quad\left[\begin{array}{l}
B_{n}^{--} R^{-n-1} \\
B_{n}^{-+} R^{-n+1}
\end{array}\right]=M_{n}(R)\left[\begin{array}{l}
B_{n}^{+-} R^{n-1} \\
B_{n}^{++} R^{n+1}
\end{array}\right] .
$$


4.3.2. Dirichlet condition on $r=R$. The aim of this subsection is to give the relation between the $n$-th component of $\varphi$ and the terms $A^{+ \pm}(n), B^{+ \pm}(n)$, and to control the other terms. For this we use the Dirichlet boundary condition $\mathbf{v}=\boldsymbol{\varphi}$ on $\partial \beta_{R}$ in (3.3). Decomposing the datum $\varphi$ and the solution $\mathbf{v}$ of (3.3) according to (3.6) and (4.1), we have

$$
c_{n}^{r}(R)=\varphi_{n}^{r}, \quad d_{n}^{r}(R)=\psi_{n}^{r}, \quad c_{n}^{\theta}(R)=\varphi_{n}^{\theta}, \quad d_{n}^{\theta}(R)=\psi_{n}^{\theta} .
$$

Using expressions (4.16)-4.17), we have

$$
\begin{aligned}
& \varphi_{n}^{r}=A_{n}^{--} R^{-n-1}+\beta_{r, n}^{-+} A_{n}^{-+} R^{-n+1}-A_{n}^{+-} R^{n-1}+\beta_{r, n}^{++} A_{n}^{++} R^{n+1}, \\
& \psi_{n}^{\theta}=A_{n}^{--} R^{-n-1}+\beta_{\theta, n}^{-+} A_{n}^{-+} R^{-n+1}+A_{n}^{+-} R^{n-1}+\beta_{\theta, n}^{++} A_{n}^{++} R^{n+1} .
\end{aligned}
$$

Using (4.46), we deduce

$$
\begin{aligned}
{\left[\begin{array}{c}
\varphi_{n}^{r} \\
\psi_{n}^{\theta}
\end{array}\right] } & =\left[\begin{array}{ll}
1 & \beta_{r, n}^{-+} \\
1 & \beta_{\theta, n}^{-+}
\end{array}\right]\left[\begin{array}{l}
A_{n}^{--} R^{-n-1} \\
A_{n}^{-+} R^{-n+1}
\end{array}\right]+\left[\begin{array}{cc}
-1 & \beta_{r, n}^{++} \\
1 & \beta_{\theta, n}^{++}
\end{array}\right]\left[\begin{array}{l}
A_{n}^{+-} R^{n-1} \\
A_{n}^{++} R^{n+1}
\end{array}\right] \\
& =M_{A}\left(\mathrm{Id}_{2}+M_{A}^{-1} N_{A} M_{n}(R)\right)\left[\begin{array}{l}
A_{n}^{+-} R^{n-1} \\
A_{n}^{++} R^{n+1}
\end{array}\right],
\end{aligned}
$$

with

$$
M_{A}=\left[\begin{array}{cc}
-1 & \beta_{r, n}^{++} \\
1 & \beta_{\theta, n}^{++}
\end{array}\right]=\left[\begin{array}{cc}
-1 & -1-\frac{2 \gamma}{n(\gamma-1)} \\
1 & 1-\frac{2}{n(\gamma-1)}
\end{array}\right], N_{A}=\left[\begin{array}{cc}
1 & \beta_{r, n}^{-+} \\
1 & \beta_{\theta, n}^{-+}
\end{array}\right]=\left[\begin{array}{cc}
1 & 1-\frac{2 \gamma}{n(\gamma-1)} \\
1 & 1+\frac{2}{n(\gamma-1)}
\end{array}\right]
$$

We notice that

$$
\operatorname{det} M_{A}=\operatorname{det} N_{A}=\frac{2(\gamma+1)}{n(\gamma-1)}, \quad M_{A}^{-1}=\frac{n(\gamma-1)}{2(\gamma+1)}\left[\begin{array}{cc}
1-\frac{2}{n(\gamma-1)} & 1+\frac{2 \gamma}{n(\gamma-1)} \\
-1 & -1
\end{array}\right] .
$$

Defining $N:=M_{A}^{-1} N_{A}$, we compute

$$
N=\frac{\gamma-1}{\gamma+1}\left[\begin{array}{cc}
n+1 & n+\frac{4 \gamma}{n(\gamma-1)^{2}} \\
-n & 1-n
\end{array}\right]
$$

Thus we have

$$
\left[\begin{array}{c}
A_{n}^{+-} R^{n-1} \\
A_{n}^{++} R^{n+1}
\end{array}\right]=\left(\operatorname{Id}_{2}+Q_{n}(R)\right)^{-1} M_{A}^{-1}\left[\begin{array}{c}
\varphi_{n}^{r} \\
\psi_{n}^{\theta}
\end{array}\right]
$$

with

$$
Q_{n}(R)=N M_{n}(R), \quad\left\|Q_{n}(R)\right\|_{\infty}=n^{2} R^{2-2 n} .
$$

Similarly, using expressions (4.18)-4.19), we have

$$
\begin{aligned}
\psi_{n}^{r} & =-B_{n}^{--} R^{-n-1}-\beta_{r, n}^{-+} B_{n}^{-+} R^{-n+1}+B_{n}^{+-} R^{n-1}-\beta_{r, n}^{++} B_{n}^{++} R^{n+1}, \\
\varphi_{n}^{\theta} & =B_{n}^{--} R^{-n-1}+\beta_{\theta, n}^{-+} B_{n}^{-+} R^{-n+1}+B_{n}^{+-} R^{n-1}+\beta_{\theta, n}^{++} B_{n}^{++} R^{n+1} .
\end{aligned}
$$

Using (4.46), we deduce

$$
\begin{aligned}
{\left[\begin{array}{c}
\psi_{n}^{r} \\
\varphi_{n}^{\theta}
\end{array}\right] } & =\left[\begin{array}{cc}
-1 & -\beta_{r, n}^{-+} \\
1 & \beta_{\theta, n}^{-+}
\end{array}\right]\left[\begin{array}{l}
B_{n}^{--} R^{-n-1} \\
B_{n}^{-+} R^{-n+1}
\end{array}\right]+\left[\begin{array}{cc}
1 & -\beta_{r, n}^{++} \\
1 & \beta_{\theta, n}^{++}
\end{array}\right]\left[\begin{array}{l}
B_{n}^{+-} R^{n-1} \\
B_{n}^{++} R^{n+1}
\end{array}\right] \\
& =M_{B}\left(\operatorname{Id}_{2}+M_{B}^{-1} N_{B} M_{n}(R)\right)\left[\begin{array}{l}
B_{n}^{+-} R^{n-1} \\
B_{n}^{++} R^{n+1}
\end{array}\right]
\end{aligned}
$$


with

$$
\begin{gathered}
M_{B}=\left[\begin{array}{cc}
1 & -\beta_{r, n}^{++} \\
1 & \beta_{\theta, n}^{++}
\end{array}\right]=\left[\begin{array}{cc}
1 & 1+\frac{2 \gamma}{n(\gamma-1)} \\
1 & 1-\frac{2}{n(\gamma-1)}
\end{array}\right], \\
N_{B}=\left[\begin{array}{cc}
-1 & -\beta_{r, n}^{-+} \\
1 & \beta_{\theta, n}^{-+}
\end{array}\right]=\left[\begin{array}{cc}
-1 & -1+\frac{2 \gamma}{n(\gamma-1)} \\
1 & 1+\frac{2}{n(\gamma-1)}
\end{array}\right] .
\end{gathered}
$$

Thus

$\operatorname{det} M_{B}=\operatorname{det} N_{B}=-\frac{2(\gamma+1)}{n(\gamma-1)}, \quad M_{B}^{-1}=-\frac{n(\gamma-1)}{2(\gamma+1)}\left[\begin{array}{cc}1-\frac{2}{n(\gamma-1)} & -1-\frac{2 \gamma}{n(\gamma-1)} \\ -1 & 1\end{array}\right]$.

We therefore have

$$
\left[\begin{array}{l}
B_{n}^{+-} R^{n-1} \\
B_{n}^{++} R^{n+1}
\end{array}\right]=\left(\operatorname{Id}_{2}+Q_{n}(R)\right)^{-1} M_{B}^{-1}\left[\begin{array}{c}
\psi_{n}^{r} \\
\varphi_{n}^{\theta}
\end{array}\right]
$$

with the same matrix $Q_{n}(R)$ defined in (4.50) for coefficients $A_{n}^{+, \pm} R^{n \pm 1}$.

Note that from (4.46), (4.49) and (4.51), we also have

$$
\left[\begin{array}{c}
A_{n}^{--} R^{-n-1} \\
A_{n}^{-+} R^{-n+1}
\end{array}\right]=M_{n}(R)\left(\operatorname{Id}_{2}+Q_{n}(R)\right)^{-1} M_{A}^{-1}\left[\begin{array}{c}
\varphi_{n}^{r} \\
\psi_{n}^{\theta}
\end{array}\right]
$$

and

$$
\left[\begin{array}{c}
B_{n}^{--} R^{-n-1} \\
B_{n}^{-+} R^{-n+1}
\end{array}\right]=M_{n}(R)\left(\operatorname{Id}_{2}+Q_{n}(R)\right)^{-1} M_{B}^{-1}\left[\begin{array}{c}
\psi_{n}^{r} \\
\varphi_{n}^{\theta}
\end{array}\right] .
$$

From (4.49) - 4.53) and expression (4.45) of $M_{n}(R)$, we get the following first rough estimate:

Lemma 4.2. There exist $R_{0}>0$ and a constant $C$ such that for any $R>R_{0}$ and any $n \geq 2$, we have with $\boldsymbol{\Phi}_{n}=\left(\varphi_{n}^{r}, \psi_{n}^{r}, \varphi_{n}^{\theta}, \psi_{n}^{\theta}\right)^{T}$ :

$$
\begin{aligned}
\left|A_{n}^{+ \pm} R^{n \pm 1}\right| & \leq C n\left\|\mathbf{\Phi}_{n}\right\|_{\infty}, \quad\left|B_{n}^{+ \pm} R^{n \pm 1}\right| \leq C n\left\|\mathbf{\Phi}_{n}\right\|_{\infty}, \\
\left|A_{n}^{- \pm} R^{-n \pm 1}\right| & \leq C\left(n^{3} R^{2-2 n}\right)\left\|\mathbf{\Phi}_{n}\right\|_{\infty}, \quad\left|B_{n}^{- \pm} R^{-n \pm 1}\right| \leq C\left(n^{3} R^{2-2 n}\right)\left\|\mathbf{\Phi}_{n}\right\|_{\infty} .
\end{aligned}
$$

4.3.3. DtN operator and Ventcel boundary condition on $r=R$. Now we are in a position to give the expression of the Dirichlet-to-Neumann operator as an operator acting on each mode $n \geq 2$ of $\boldsymbol{\varphi}$, and exhibit Ventcel boundary condition (3.5) in mode $n$ as in Proposition 3.2 .

Recall that $\Lambda_{R}(\boldsymbol{\varphi})=\frac{R(1+\nu)}{E} \sigma(\mathbf{v}) \cdot \mathbf{n}_{\mid r=R}$. We use (4.36), with the explicit components computed in (4.37)-(4.40). The first component for mode $n$ of $\Lambda_{R}(\varphi)$ is then

$$
\begin{gathered}
{\left[\begin{array}{c}
1-n \\
\frac{2}{n}+1-n
\end{array}\right]^{\top}\left(\left[\begin{array}{c}
A_{n}^{+-} R^{n-1} \\
A_{n}^{++} R^{n+1}
\end{array}\right] \cos n \theta-\left[\begin{array}{l}
B_{n}^{+-} R^{n-1} \\
B_{n}^{++} R^{n+1}
\end{array}\right] \sin n \theta\right)} \\
+\left[\begin{array}{c}
-n-1 \\
\frac{2}{n}-1-n
\end{array}\right]^{\top}\left(\left[\begin{array}{c}
A_{n}^{--} R^{-n-1} \\
A_{n}^{-+} R^{-n+1}
\end{array}\right] \cos n \theta-\left[\begin{array}{l}
B_{n}^{--} R^{-n-1} \\
B_{n}^{-+} R^{-n+1}
\end{array}\right] \sin n \theta\right) \\
=\left(\left[\begin{array}{c}
1-n \\
\frac{2}{n}+1-n
\end{array}\right]^{\top}+\left[\begin{array}{c}
-n-1 \\
\frac{2}{n}-1-n
\end{array}\right]^{\top} M_{n}(R)\right) \\
\times\left(\left[\begin{array}{l}
A_{n}^{+-} R^{n-1} \\
A_{n}^{++} R^{n+1}
\end{array}\right] \cos n \theta-\left[\begin{array}{l}
B_{n}^{+-} R^{n-1} \\
B_{n}^{++} R^{n+1}
\end{array}\right] \sin n \theta\right)
\end{gathered}
$$




$$
\begin{aligned}
=\left(\left[\begin{array}{c}
1-n \\
\frac{2}{n}+1-n
\end{array}\right]^{\top}+\left[\begin{array}{c}
-n-1 \\
\frac{2}{n}-1-n
\end{array}\right]^{\top} M_{n}(R)\right) \\
\quad \times\left(\operatorname{Id}_{2}+Q_{n}(R)\right)^{-1}\left(M_{A}^{-1}\left[\begin{array}{c}
\varphi_{n}^{r} \\
\psi_{n}^{\theta}
\end{array}\right] \cos n \theta-M_{B}^{-1}\left[\begin{array}{c}
\psi_{n}^{r} \\
\varphi_{n}^{\theta}
\end{array}\right] \sin n \theta\right) .
\end{aligned}
$$

Let us now deal with the second component in mode $n \geq 2$ of $\Lambda_{R}(\boldsymbol{\varphi})$. This is

$$
\begin{aligned}
& {\left[\begin{array}{l}
n-1 \\
n+1
\end{array}\right]^{\top}\left(\left[\begin{array}{l}
B_{n}^{+-} R^{n-1} \\
B_{n}^{++} R^{n+1}
\end{array}\right] \cos n \theta+\left[\begin{array}{l}
A_{n}^{+-} R^{n-1} \\
A_{n}^{++} R^{n+1}
\end{array}\right] \sin n \theta\right)} \\
& +\left[\begin{array}{c}
-n-1 \\
1-n
\end{array}\right]^{\top}\left(\left[\begin{array}{l}
B_{n}^{--} R^{-n-1} \\
B_{n}^{-+} R^{-n+1}
\end{array}\right] \cos n \theta+\left[\begin{array}{l}
A_{n}^{--} R^{-n-1} \\
A_{n}^{-+} R^{-n+1}
\end{array}\right] \sin n \theta\right) \\
& =\left(\left[\begin{array}{l}
n-1 \\
n+1
\end{array}\right]^{\top}+\left[\begin{array}{c}
-n-1 \\
1-n
\end{array}\right]^{\top} M_{n}(R)\right)\left(\left[\begin{array}{l}
B_{n}^{+-} R^{n-1} \\
B_{n}^{++} R^{n+1}
\end{array}\right] \cos n \theta+\left[\begin{array}{l}
A_{n}^{+-} R^{n-1} \\
A_{n}^{++} R^{n+1}
\end{array}\right] \sin n \theta\right) \\
& (4.55)=\left(\left[\begin{array}{l}
n-1 \\
n+1
\end{array}\right]^{\top}+\left[\begin{array}{c}
-n-1 \\
1-n
\end{array}\right]^{\top} M_{n}(R)\right) \\
& \times\left(\operatorname{Id}_{2}+Q_{n}(R)\right)^{-1}\left(M_{B}^{-1}\left[\begin{array}{c}
\psi_{n}^{r} \\
\varphi_{n}^{\theta}
\end{array}\right] \cos n \theta+M_{A}^{-1}\left[\begin{array}{c}
\varphi_{n}^{r} \\
\psi_{n}^{\theta}
\end{array}\right] \sin n \theta\right) .
\end{aligned}
$$

Let us denote

$$
V_{1}=\left[\begin{array}{c}
1-n \\
\frac{2}{n}+1-n
\end{array}\right] \quad \text { and } \quad V_{2}=\left[\begin{array}{l}
n-1 \\
n+1
\end{array}\right] .
$$

Thus a simple computation gives

$$
\begin{aligned}
V_{1}^{\top} M_{A}^{-1} & =\frac{1}{1+\gamma}[n-\gamma, 1-n \gamma], & V_{1}^{\top} M_{B}^{-1} & =\frac{1}{1+\gamma}[-(n-\gamma), 1-n \gamma], \\
V_{2}^{\top} M_{A}^{-1} & =\frac{1}{1+\gamma}[1-n \gamma, n-\gamma], & V_{2}^{\top} M_{B}^{-1} & =\frac{1}{1+\gamma}[-(1-n \gamma), n-\gamma] .
\end{aligned}
$$

The main term for mode $n \geq 2$ for the Dirichlet-to-Neumann operator is then

$$
\frac{1}{1+\gamma}\left[\begin{array}{l}
\cos n \theta\left((n-\gamma) \varphi_{n}^{r}+(1-n \gamma) \psi_{n}^{\theta}\right)+\sin n \theta\left((n-\gamma) \psi_{n}^{r}+(n \gamma-1) \varphi_{n}^{\theta}\right) \\
\cos n \theta\left((n \gamma-1) \psi_{n}^{r}+(n-\gamma) \varphi_{n}^{\theta}\right)+\sin n \theta\left((1-n \gamma) \varphi_{n}^{r}+(n-\gamma) \psi_{n}^{\theta}\right)
\end{array}\right] .
$$

Defining for each $n \geq 2$ the vector $\boldsymbol{\Phi}_{n}=\left(\varphi_{n}^{r}, \psi_{n}^{r}, \varphi_{n}^{\theta}, \psi_{n}^{\theta}\right)^{\top}$, we get that in the corresponding basis, the matrix for mode $n$ of $\Lambda_{R}(\varphi)$ is given of the form $\Lambda_{n}+\mathcal{R}_{n, R}$, with

$$
\Lambda_{n}=\frac{1}{1+\gamma}\left[\begin{array}{cccc}
n-\gamma & 0 & 0 & 1-n \gamma \\
0 & n-\gamma & n \gamma-1 & 0 \\
0 & n \gamma-1 & n-\gamma & 0 \\
1-n \gamma & 0 & 0 & n-\gamma
\end{array}\right]
$$

From (4.55) and expressions (4.45) and (4.50) of matrices $M_{n}(R)$ and $Q_{n}(R)$, we get

$$
\left\|\mathcal{R}_{n, R}\right\|_{\infty} \leq C_{\gamma} n^{2} R^{-2 n+2},
$$

for a fixed constant $C_{\gamma}$. 
We can now give the expression of the Ventcel boundary condition in mode $n$ as a matrix in the same basis. Using decomposition (3.6) and relation (4.6), we can rewrite the left-hand side of (3.5) in the form $\left(P_{n}+\mathcal{R}_{n, R}\right) \boldsymbol{\Phi}_{n}$, with $P_{n}$ given by

$$
\begin{array}{r}
P_{n}=\frac{-n^{2}}{4}\left[\begin{array}{cccc}
\frac{\nu}{1-\nu} & 0 & 0 & 0 \\
0 & \frac{\nu}{1-\nu} & 0 & 0 \\
0 & 0 & \frac{2(1-\nu)}{1-2 \nu} & 0 \\
0 & 0 & 0 & \frac{2(1-\nu)}{1-2 \nu}
\end{array}\right]+\operatorname{Id}_{4} \\
+\frac{1}{1+\gamma}\left[\begin{array}{cccc}
n-\gamma & 0 & 0 & 1-n \gamma \\
0 & n-\gamma & n \gamma-1 & 0 \\
0 & n \gamma-1 & n-\gamma & 0 \\
1-n \gamma & 0 & 0 & n-\gamma
\end{array}\right]
\end{array}
$$

Using (4.6), we have

$$
\nu=\frac{1-2 \gamma}{2(1-\gamma)}, \quad \frac{\nu}{1-\nu}=1-2 \gamma, \quad \frac{1-\nu}{1-2 \nu}=\frac{1}{2 \gamma},
$$

so that $P_{n}$ can be written in the following form:

$$
\begin{aligned}
P_{n}=\frac{-n^{2}}{4}\left[\begin{array}{cccc}
1-2 \gamma & 0 & 0 & 0 \\
0 & 1-2 \gamma & 0 & 0 \\
0 & 0 & \frac{1}{\gamma} & 0 \\
0 & 0 & 0 & \frac{1}{\gamma}
\end{array}\right]+\operatorname{Id}_{4} \\
+\frac{1}{1+\gamma}\left[\begin{array}{cccc}
n-\gamma & 0 & 0 & 1-n \gamma \\
0 & n-\gamma & n \gamma-1 & 0 \\
0 & n \gamma-1 & n-\gamma & 0 \\
1-n \gamma & 0 & 0 & n-\gamma
\end{array}\right]
\end{aligned}
$$

This completes the proof of Proposition 3.2 in case $n \geq 2$.

4.4. DtN map and Ventcel conditions for modes $n=1,0$. We now follow the same strategy for the mode $n=1$ and the constant term $n=0$ in (4.36).

4.4.1. Case $n=1$. The first step is to use Neumann conditions on the inner circle of radius $r=1$ :

$$
\left.\frac{1+\nu}{E} r \sigma(\mathbf{v}) \cdot \mathbf{n}\right|_{r=1}=0 .
$$

This implies that for $n=1, r=1$,

$$
c_{1}(n, r)=s_{2}(n, r)=0 \quad \text { and } \quad s_{1}(n, r)=c_{2}(n, r)=0 .
$$

Using (4.41) we directly get

$$
A_{n}^{--}=A_{n}^{++} \quad \text { and } \quad B_{n}^{--}=B_{n}^{++} \quad \text { when } n=1,
$$

so that

$$
A_{n}^{--} R^{-2}=R^{-4} A_{n}^{++} R^{2}, \quad B_{n}^{--} R^{-2}=R^{-4} B_{n}^{++^{2}} \quad \text { when } n=1 .
$$

Now we apply the Dirichlet condition in (3.3) on $r=R$, which reads

$$
c_{n}^{r}(R)=\varphi_{n}^{r}, \quad d_{n}^{r}(R)=\psi_{n}^{r}, \quad c_{n}^{\theta}(R)=\varphi_{n}^{\theta}, \quad d_{n}^{\theta}(R)=\psi_{n}^{\theta} .
$$


Using (4.21)-4.24, we get

$$
\begin{aligned}
\varphi_{n}^{r} & =A_{n}^{--} R^{-2}-A_{n}^{00}+\beta_{r, n}^{++} A_{n}^{++} R^{2}, \\
\psi_{n}^{\theta} & =A_{n}^{-}-R^{-2}+A_{n}^{00}+\beta_{\theta, n}^{++} A_{n}^{++} R^{2}, \\
\psi_{n}^{r} & =-B_{n}^{--} R^{-2}+B_{n}^{00}-\beta_{r, n}^{++} B_{n}^{++} R^{2}, \\
\varphi_{n}^{\theta} & =B_{n}^{--} R^{-2}+B_{n}^{00}+\beta_{\theta, n}^{++} B_{n}^{++} R^{2} .
\end{aligned}
$$

Adding the first two equalites and substracting the last ones give:

$$
\begin{aligned}
\varphi_{n}^{r}+\psi_{n}^{\theta} & =2 A_{n}^{--} R^{-2}+S^{++} A_{n}^{++} R^{2}, \\
\varphi_{n}^{\theta}-\psi_{n}^{r} & =2 B_{n}^{--} R^{-2}+S^{++} B_{n}^{++} R^{2},
\end{aligned}
$$

with

$$
S^{++}=\beta_{r, n}^{++}+\beta_{\theta, n}^{++}=\frac{-2(\gamma+1)}{n(\gamma-1)} .
$$

Using (4.58), we deduce

$$
\begin{aligned}
& \varphi_{n}^{r}+\psi_{n}^{\theta}=S^{++}\left(1+2\left(S^{++}\right)^{-1} R^{-4}\right) A_{n}^{++} R^{2}, \\
& \varphi_{n}^{\theta}-\psi_{n}^{r}=S^{++}\left(1+2\left(S^{++}\right)^{-1} R^{-4}\right) B_{n}^{++} R^{2},
\end{aligned}
$$

so that denoting the scalar $Q_{1}(R)=2\left(S^{++}\right)^{-1} R^{-4}$, we can write

$$
\begin{aligned}
& A_{n}^{++} R^{2}=\left(S^{++}\left(1+Q_{1}(R)\right)\right)^{-1}\left(\varphi_{n}^{r}+\psi_{n}^{\theta}\right), \\
& B_{n}^{++} R^{2}=\left(S^{++}\left(1+Q_{1}(R)\right)\right)^{-1}\left(\varphi_{n}^{\theta}-\psi_{n}^{r}\right) .
\end{aligned}
$$

Note that, using (4.58), we get in that case

$$
\begin{aligned}
& A_{n}^{--} R^{-2}=R^{-4}\left(1+Q_{n}(R)\right)^{-1}\left(S^{++}\right)^{-1}\left(\varphi_{n}^{r}+\psi_{n}^{\theta}\right), \\
& B_{n}^{--} R^{-2}=R^{-4}\left(1+Q_{n}(R)\right)^{-1}\left(S^{++}\right)^{-1}\left(\varphi_{n}^{\theta}-\psi_{n}^{r}\right) .
\end{aligned}
$$

We therefore have proven the following lemma:

Lemma 4.3. There exist $R_{0}>0$ and a constant $C$ such that for any $R>R_{0}$ and $n=1$, we have with $\boldsymbol{\Phi}_{n}=\left(\varphi_{n}^{r}, \psi_{n}^{r}, \varphi_{n}^{\theta}, \psi_{n}^{\theta}\right)^{T}$ :

$$
\begin{aligned}
\left|A_{n}^{++} R^{2}\right| & \leq C\left\|\boldsymbol{\Phi}_{n}\right\|_{\infty}, & \left|B_{n}^{++} R^{2}\right| & \leq C\left\|\boldsymbol{\Phi}_{n}\right\|_{\infty}, \\
\left|A_{n}^{--} R^{-2}\right| & \leq C R^{-4}\left\|\boldsymbol{\Phi}_{n}\right\|_{\infty}, & \left|B_{n}^{- \pm} R^{-2}\right| & \leq R^{-4}\left\|\boldsymbol{\Phi}_{n}\right\|_{\infty} .
\end{aligned}
$$

Note that we don't have any control on $A_{n}^{00}$. It will appear later that it is not necessary.

Now we focus on what happens on the circle $r=R$. We give the expression for mode $n=1$ of the components of $\Lambda_{R}(\boldsymbol{\varphi})=\frac{R(1+\nu)}{E} \sigma(\mathbf{v}) \cdot \mathbf{n}_{\mid r=R}$. We use (4.36), with the explicit components computed in (4.41). The first component for mode $n=1$ of $\Lambda_{R}(\varphi)$ is therefore

$$
\begin{aligned}
& \frac{1}{2 \gamma}\left(c_{1}(n, r) \cos \theta+s_{1}(n, r) \sin \theta\right) \\
& =2\left(\left(-A_{n}^{--} R^{-2}+A_{n}^{++} R^{2}\right) \cos \theta+\left(B_{n}^{--} R^{-2}-B_{n}^{++} R^{2}\right) \sin \theta\right) \\
& =2\left(S^{++}\right)^{-1}\left(1-R^{-4}\right)\left(1+Q_{1}(R)\right)^{-1}\left(\left(\varphi_{n}^{r}+\psi_{n}^{\theta}\right) \cos \theta+\left(\psi_{n}^{r}-\varphi_{n}^{\theta}\right) \sin \theta\right),
\end{aligned}
$$

where we used (4.58) and (4.61) for the last equality. An easy computation gives

$$
2\left(S^{++}\right)^{-1}=\frac{1-\gamma}{1+\gamma},
$$


so that the first component for mode 1 of $\Lambda_{R}(\varphi)$ is given by

$$
\frac{1-\gamma}{1+\gamma}\left(1-R^{-4}\right)\left(1+Q_{1}(R)\right)^{-1}\left(\left(\varphi_{n}^{r}+\psi_{n}^{\theta}\right) \cos \theta+\left(\psi_{n}^{r}-\varphi_{n}^{\theta}\right) \sin \theta\right) .
$$

Similarly, the second component for mode 1 of $\Lambda_{R}(\varphi)$ is given by

$$
2\left(\left(-B_{n}^{--} R^{-2}+B_{n}^{++} R^{2}\right) \cos \theta+\left(-A_{n}^{--} R^{-2}+A_{n}^{++} R^{2}\right) \sin \theta\right),
$$

which turns out to be equal to

$$
\frac{1-\gamma}{1+\gamma}\left(1+Q_{1}(R)\right)^{-1}\left(\left(\varphi_{n}^{\theta}-\psi_{n}^{r}\right) \cos \theta+\left(\varphi_{n}^{r}+\psi_{n}^{\theta}\right) \sin \theta\right) .
$$

Using the vector $\boldsymbol{\Phi}_{n}=\left(\varphi_{n}^{r}, \psi_{n}^{r}, \varphi_{n}^{\theta}, \psi_{n}^{\theta}\right)^{\top}$ for $n=1$, we get that the matrix of $\Lambda_{R}(\boldsymbol{\varphi})$ for mode $n=1$ in this basis is of the form $\Lambda_{n}+\mathcal{R}_{n, R}$ with

$$
\Lambda_{n}=\frac{1}{1+\gamma}\left[\begin{array}{cccc}
1-\gamma & 0 & 0 & 1-\gamma \\
0 & 1-\gamma & \gamma-1 & 0 \\
0 & \gamma-1 & 1-\gamma & 0 \\
1-\gamma & 0 & 0 & 1-\gamma
\end{array}\right]
$$

From (4.63) and (4.64), and the expression of matrix $Q_{n}(R)$, we get that for $n=1$,

$$
\left|\mathcal{R}_{n, R}\right| \leq C_{\gamma} R^{-4}
$$

for a fixed constant $C_{\gamma}$. Note that this coincides with the general expression for $n \geq 2$ given in (4.57). As a direct consequence, we get the same result as in case $n \geq 2$ : Using decomposition (3.6), we can rewrite the left-hand-side of (3.5) for mode $n=1$ in the form $\left(P_{n}+\mathcal{R}_{n, R}\right) \boldsymbol{\Phi}_{n}$, with $P_{n}$ given by

$$
\begin{aligned}
P_{n}=\frac{-n^{2}}{4}\left[\begin{array}{cccc}
1-2 \gamma & 0 & 0 & 0 \\
0 & 1-2 \gamma & 0 & 0 \\
0 & 0 & \frac{1}{\gamma} & 0 \\
0 & 0 & 0 & \frac{1}{\gamma}
\end{array}\right]+\operatorname{Id}_{4} \\
+\frac{1}{1+\gamma}\left[\begin{array}{cccc}
n-\gamma & 0 & 0 & 1-n \gamma \\
0 & n-\gamma & n \gamma-1 & 0 \\
0 & n \gamma-1 & n-\gamma & 0 \\
1-n \gamma & 0 & 0 & n-\gamma
\end{array}\right]
\end{aligned}
$$

This completes the proof of Proposition 3.2 in case $n=1$.

4.4.2. Case $n=0$. We follow the same strategy in this much simpler case. We first use Neumann conditions on the inner circle of radius $r=1$ :

$$
\left.\frac{1+\nu}{E} r \sigma(\mathbf{v}) \cdot \mathbf{n}\right|_{r=1}=0 \text {. }
$$

Using (4.36), this implies that

$$
c_{1}(r)=0 \quad \text { and } \quad c_{2}(r)=0 .
$$

Using (4.42), we directly get

$$
\gamma A_{0}^{--}=(1-\gamma) A_{0}^{++}, \quad B_{0}^{--}=0 .
$$

This can be rewritten as

$$
A_{0}^{--} R^{-1}=\frac{1-\gamma}{\gamma} R^{-2} A_{0}^{++} R, \quad B_{0}^{--} R^{-1}=0 .
$$


Now we apply the Dirichlet condition in (3.3) on $r=R$, which tells us that

$$
e_{0}^{r}(R)=\varphi_{0}^{r}, \quad e_{0}^{\theta}(R)=\varphi_{0}^{\theta} .
$$

Using (4.28)-(4.29) we get

$$
\varphi_{0}^{r}=A_{0}^{--} R^{-1}+A_{0}^{++} R, \quad \varphi_{0}^{\theta}=B_{0}^{--} R^{-1}+B_{0}^{++} R .
$$

Using (4.66) we therefore get

$$
\varphi_{0}^{r}=\left(1+\frac{1-\gamma}{\gamma} R^{-2}\right) A_{0}^{++} R, \quad \varphi_{0}^{\theta}=B_{0}^{++} R,
$$

and therefore

$$
A_{0}^{++} R=\left(1+\frac{1-\gamma}{\gamma} R^{-2}\right)^{-1} \varphi_{0}^{r}, \quad B_{0}^{++} R=\varphi_{0}^{\theta} .
$$

Thus we have proven the following lemma.

Lemma 4.4. There exist $R_{0}>0$ and a constant $C$ such that for any $R>R_{0}$, we have with $\boldsymbol{\Phi}_{0}=\left(\varphi_{0}^{r}, \varphi_{0}^{\theta}\right)^{T}$ :

$$
\begin{aligned}
\left|A_{0}^{++} R\right| & \leq C\left\|\boldsymbol{\Phi}_{0}\right\|_{\infty}, & \left|B_{0}^{++} R\right| & \leq C\left\|\Phi_{0}\right\|_{\infty}, \\
\left|A_{0}^{--} R^{-1}\right| & \leq C R^{-2}\left\|\boldsymbol{\Phi}_{0}\right\|_{\infty}, & B_{0}^{--} R^{-2} & =0 .
\end{aligned}
$$

Now we look at what happens on the circle $r=R$. We give the expression for mode $n=0$ of the components of $\Lambda_{R}(\boldsymbol{\varphi})=\frac{R(1+\nu)}{E} \sigma(\mathbf{v}) \cdot \mathbf{n}_{\mid r=R}$. We use (4.36), with the explicit expressions computed in (4.42). The components for mode $n=0$ of $\Lambda_{R}(\varphi)$ are (for $r=R$ )

$$
\left[\begin{array}{c}
\frac{1}{2 \gamma} c_{1}(r) \\
\frac{1}{2} c_{2}(r)
\end{array}\right]=\left[\begin{array}{c}
\frac{1}{2 \gamma}\left(-2 \gamma A_{0}^{--} R^{-1}+2(1-\gamma) A_{0}^{++} R\right) \\
\frac{1}{2}\left(-2 B_{0}^{--} R^{-1}\right)
\end{array}\right] .
$$

Using (4.66) and (4.67), we get that for $r=R$,

$$
\begin{aligned}
{\left[\begin{array}{c}
\frac{1}{2 \gamma} c_{1}(r) \\
\frac{1}{2} c_{2}(r)
\end{array}\right] } & =\left[\begin{array}{c}
\frac{1-\gamma}{\gamma}\left(1-R^{-2}\right) A_{0}^{++} R \\
0
\end{array}\right] \\
& =\left[\begin{array}{c}
\frac{1-\gamma}{\gamma}\left(1-R^{-2}\right)\left(1+\frac{1-\gamma}{\gamma} R^{-2}\right)^{-1} \varphi_{0}^{r} \\
0
\end{array}\right] .
\end{aligned}
$$

We now introduce the vector $\boldsymbol{\Phi}_{0}=\left(\varphi_{0}^{r}, \varphi_{0}^{\theta},\right)^{\top}$. With respect to this decomposition, we get that the matrix of $\Lambda_{R}(\varphi)$ for mode $n=0$ is of the form $\Lambda_{n}+\mathcal{R}_{0}$ with

$$
\Lambda_{0}=\left[\begin{array}{cc}
\frac{1-\gamma}{\gamma} & 0 \\
0 & 0
\end{array}\right] \quad \text { and } \quad\left\|\mathcal{R}_{0}\right\|_{\infty} \leq C R^{-2}
$$

Using decomposition (3.6), we can rewrite the left-hand-side of (3.5) for mode $n=0$ in the form $\left(P_{n}+\mathcal{R}_{n, R}\right) \boldsymbol{\Phi}_{n}$, with $P_{n}$ given by

$$
P_{0}=\operatorname{Id}_{2}+\left[\begin{array}{cc}
\frac{1-\gamma}{\gamma} & 0 \\
0 & 0
\end{array}\right]=\left[\begin{array}{cc}
\frac{1}{\gamma} & 0 \\
0 & 1
\end{array}\right]
$$

This completes the proof of Proposition 3.2 in case $n=0$. 

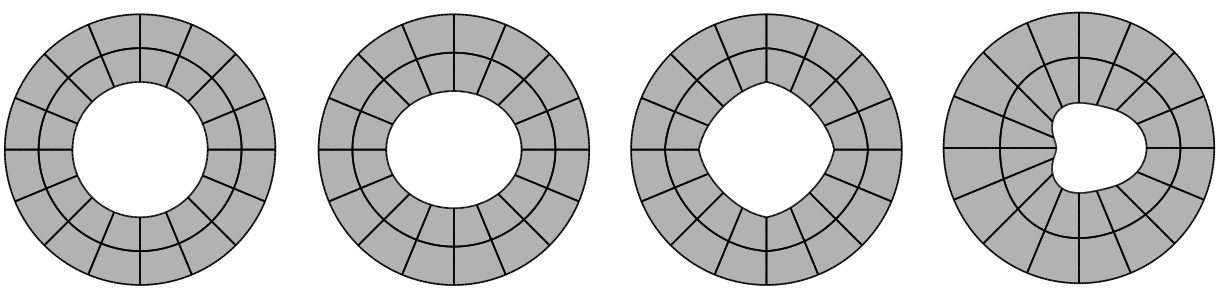

Figure 2. Disk, ellipse with eccentricity 0.5, "generic" domains $\left(R=2\right.$ and $\mathbb{Q}_{6}$ meshes $)$.

\section{Numerical RESUlts}

5.1. Fixed parameters - various geometries. In this section, we have set the mechanical paramaters as follows:

$$
\lambda=0.5769230769, \quad \mu=0.3846153396 .
$$

We consider problem (1.4) for various interior domains $\omega$; see Figure 2 The exterior radius $R$ varies. In order to detect the forbidden values of $R$, we investigate the norm of the inverse of the operator $L_{R}$ associated with problem (1.4).

The finite element resolution is performed using the library Mélina [29], with isoparametric $\mathbb{Q}_{6}$ lagrangian elements (the mesh for the presented results is made up of 16 such curvilinear elements). In Figure 3, we have plotted the norm of the inverse of the operator $L_{R}$ associated with problem (1.4) with respect to the external radius $R$ (in logarithmic coordinates) in the case where $\omega$ is the unit disk or an ellipse close to this disk. It turns out that, as expected, no forbidden ratio is encountered for "large" values of $R$. A zoom near the small values of $R$ is shown in the left plot, where several singular radii are present. The forbidden radii are close to each other for the first two cases, which is in accordance with the perturbation arguments developed in Subsection 3.4. In Figure 4, we show the dependence of the forbidden radii with respect to the eccentricity of the ellipse.

In Figure 5] we present the same results for more generic geometry: the third and fourth domains plotted in Figure 2
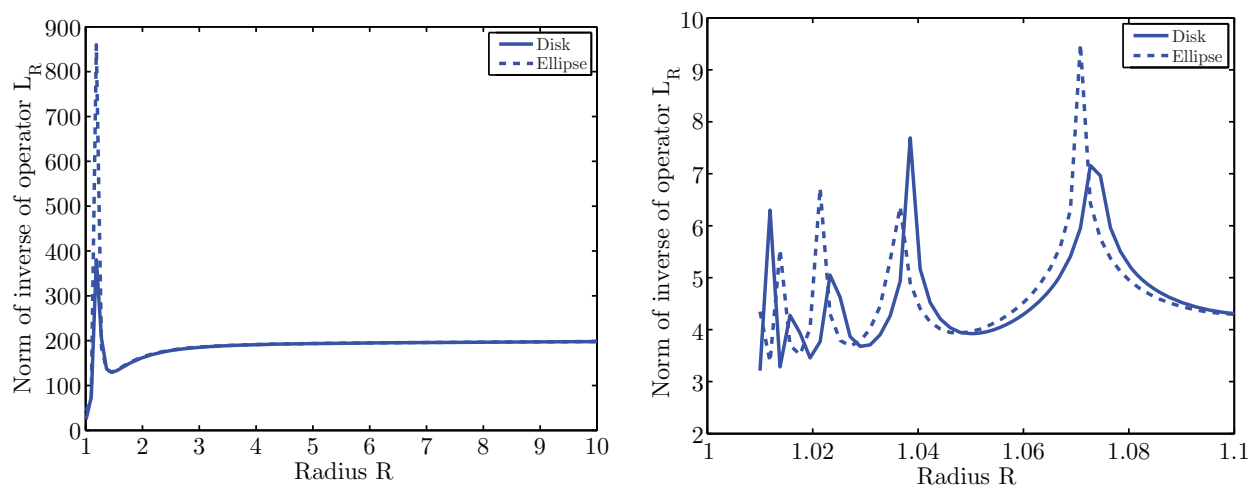

FiguRE 3. Norm of resolvent for the disk and for an "almost disk". (Color available online.) 


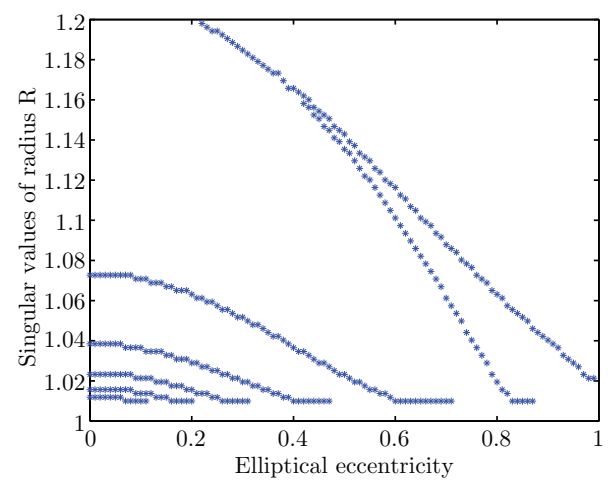

Figure 4. Dependence of singular values of $R$ for an ellipse $\omega$ with respect to its eccentricity. (Color available online.)
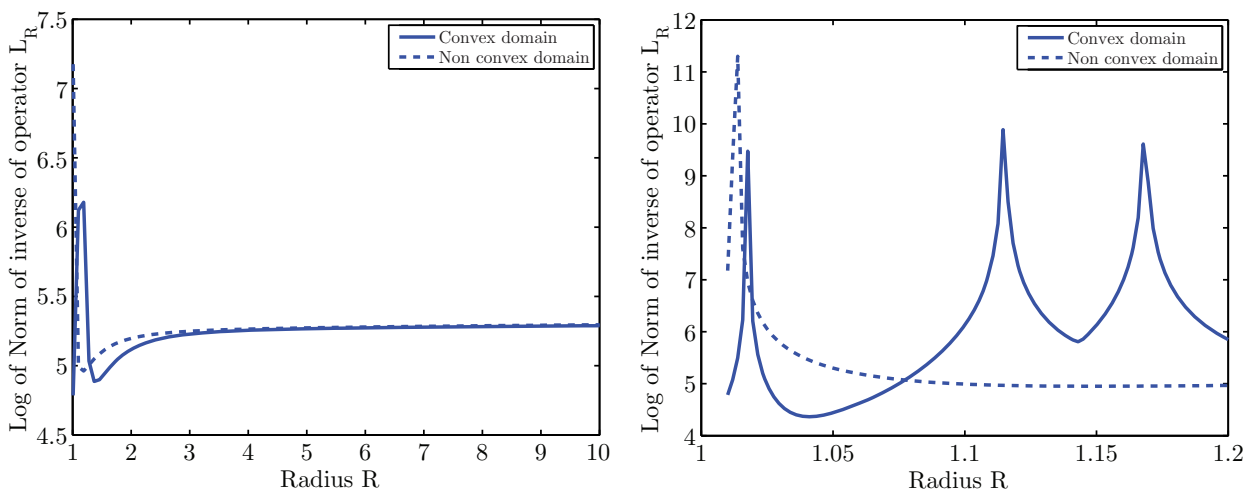

Figure 5. Norm of resolvent for the "generic" domains of Figure2 $(\nu=0.5)$. (Color available online. $)$
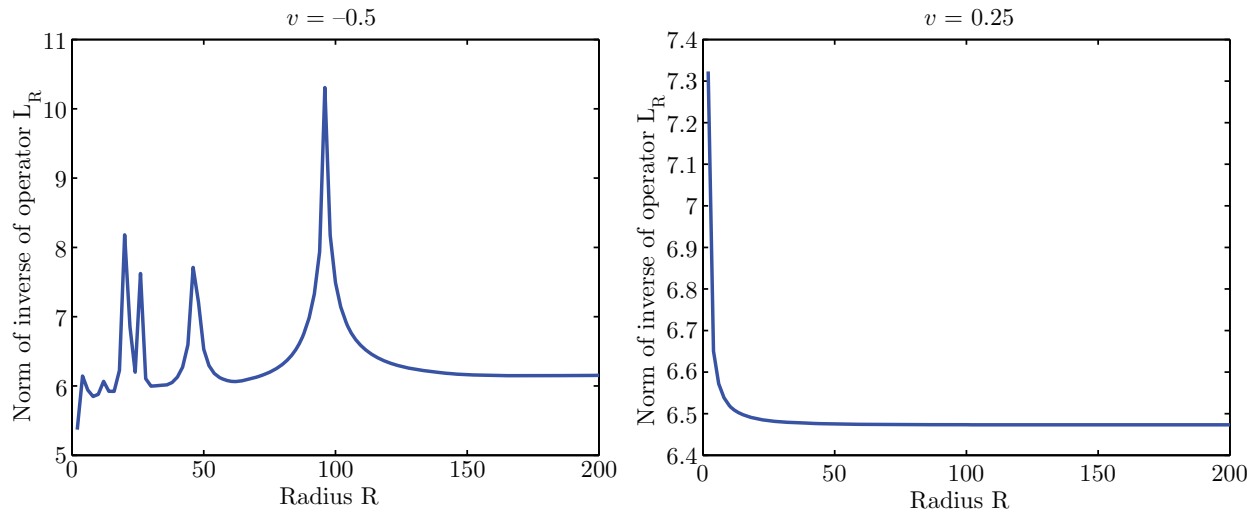

Figure 6. Norm of resolvent for a disk and different values $\nu$. (Color available online.) 
5.2. Fixed geometry - moving parameters. In this part, we fix the Young modulus: $E=1$, and the Poisson ratio $\nu$ is varying. We present the results obtained for two values of $\nu$ in Figure 6. Let us mention that more forbidden radii are observed for $\nu<0$ than for $\nu>0$.

\section{ACKNowledgements}

First reflections about this work were supported by the ANR (Agence Nationale de la Recherche), projects MACADAM no JCJC06-139561 and ARAMIS n ${ }^{\circ} 12$ BS010021. The authors also thank the Erwin Schrödinger Institute and the MittagLeffler Institute for their hospitality and very good working conditions.

\section{REFERENCES}

[1] Y. Achdou, O. Pironneau, and F. Valentin, Effective boundary conditions for laminar flows over periodic rough boundaries, J. Comput. Phys. 147 (1998), no. 1, 187-218, DOI 10.1006/jcph.1998.6088. MR.1657773(99j:76086)

[2] Y. Amirat, G. A. Chechkin, and R. R. Gadyl'shin, Asymptotics of simple eigenvalues and eigenfunctions for the Laplace operator in a domain with oscillating boundary (English, with Russian summary), Zh. Vychisl. Mat. Mat. Fiz. 46 (2006), no. 1, 102-115, DOI 10.1134/S0965542506010118; English transl., Comput. Math. Math. Phys. 46 (2006), no. 1, 97-110. MR2239730(2007d:35009)

[3] H. Ammari, E. Bonnetier, Y. Capdeboscq, M. Tanter, and M. Fink, Electrical impedance tomography by elastic deformation, SIAM J. Appl. Math. 68 (2008), no. 6, 1557-1573, DOI 10.1137/070686408. MR2424952 (2009h:35439)

[4] H. Ammari, H. Kang, M. Lim, and H. Zribi, Layer potential techniques in spectral analysis. Part I: Complete asymptotic expansions for eigenvalues of the Laplacian in domains with small inclusions, Trans. Amer. Math. Soc. 362 (2010), no. 6, 2901-2922, DOI 10.1090/S00029947-10-04695-7. MR2592941 (2012c:35078)

[5] M. F. Ben Hassen and E. Bonnetier, An asymptotic formula for the voltage potential in a perturbed $\epsilon$-periodic composite medium containing misplaced inclusions of size $\epsilon$, Proc. Roy. Soc. Edinburgh Sect. A 136 (2006), no. 4, 669-700, DOI 10.1017/S0308210500004650. MR2250439 (2007f:35015)

[6] A. Bendali and K. Lemrabet, The effect of a thin coating on the scattering of a time-harmonic wave for the Helmholtz equation, SIAM J. Appl. Math. 56 (1996), no. 6, 1664-1693, DOI 10.1137/S0036139995281822. MR1417476 (97i:78009)

[7] P. Bettess, Infinite Elements, Penshaw Press, Paris, 1992.

[8] V. Bonnaillie-Noël, D. Brancherie, M. Dambrine, F. Hérau, S. Tordeux, and G. Vial, Multiscale expansion and numerical approximation for surface defects (English, with English and French summaries), CANUM 2010, 40 Congrès National d'Analyse Numérique, ESAIM Proc., vol. 33, EDP Sci., Les Ulis, 2011, pp. 22-35, DOI 10.1051/proc/201133003. MR2863307 (2012m:35010)

[9] V. Bonnaillie-Noël, M. Dambrine, F. Hérau, and G. Vial, On generalized Ventcel's type boundary conditions for Laplace operator in a bounded domain, SIAM J. Math. Anal. 42 (2010), no. 2, 931-945, DOI 10.1137/090756521. MR2644364(2011k:35035)

[10] V. Bonnaillie-Noël, M. Dambrine, S. Tordeux, and G. Vial, Interactions between moderately close inclusions for the Laplace equation, Math. Models Methods Appl. Sci. 19 (2009), no. 10, 1853-1882, DOI 10.1142/S021820250900398X. MR2573145(2011a:35023)

[11] B. Bourdin, G. A. Francfort, and J.-J. Marigo, The Variational Approach to Fracture, Springer, New York, 2008. Reprinted from J. Elasticity 91 (2008), no. 1-3 [MR2390547]. With a foreword by Roger Fosdick. MR2473620 (2009m:49017)

[12] D. Bresch and V. Milisic, High order multi-scale wall-laws, Part I: the periodic case, Quart. Appl. Math. 68 (2010), no. 2, 229-253. MR.2663000(2011k:76029)

[13] M. Dauge, Elliptic Boundary Value Problems on Corner Domains, Lecture Notes in Mathematics, vol. 1341, Springer-Verlag, Berlin, 1988. Smoothness and asymptotics of solutions. MR.961439(91a:35078) 
[14] B. Delourme, Modèles et asymptotiques des interfaces fines et périodiques, Ph.D. thesis, Université Pierre et Marie Curie, Paris VI, 2010.

[15] B. Delourme, H. Haddar, and P. Joly, Approximate models for wave propagation across thin periodic interfaces (English, with English and French summaries), J. Math. Pures Appl. (9) 98 (2012), no. 1, 28-71, DOI 10.1016/j.matpur.2012.01.003. MR.2935369

[16] B. Engquist and A. Majda, Absorbing boundary conditions for the numerical simulation of waves, Math. Comp. 31 (1977), no. 139, 629-651. MR0436612 (55 \#9555)

[17] B. Engquist and J. C. Nedelec, Effective boundary conditions for electromagnetic scattering in thin layers, Rapport interne 278, CMAP, 1993.

[18] D. Givoli, Nonreflecting boundary conditions, J. Comput. Phys. 94 (1991), no. 1, 1-29, DOI 10.1016/0021-9991(91)90135-8. MR1103713(92a:65266)

[19] P. Grisvard, Boundary Value Problems in Non-Smooth Domains, Pitman, London, 1985.

[20] P. Grisvard, Problèmes aux limites dans les polygones. Mode d'emploi (French), EDF Bull. Direction Études Rech. Sér. C Math. Inform. 1 (1986), 3, 21-59. MR840970 (87g:35073)

[21] P. Grisvard, Singularités en elasticité (French, with English summary), Arch. Rational Mech. Anal. 107 (1989), no. 2, 157-180, DOI 10.1007/BF00286498. MR996909 (90j:35170)

[22] H. Haddar and P. Joly, Effective boundary conditions for thin ferromagnetic coatings. Asymptotic analysis of the $1 D$ model, Asymptot. Anal. 27 (2001), no. 2, 127-160. MR.1852003 (2002g:78036)

[23] L. Halpern and J. Rauch, Absorbing boundary conditions for diffusion equations (English, with English and French summaries), Numer. Math. 71 (1995), no. 2, 185-224, DOI 10.1007/s002110050141. MR 1347164(96h:65152)

[24] W. Jäger and A. Mikelić, On the roughness-induced effective boundary conditions for an incompressible viscous flow, J. Differential Equations 170 (2001), no. 1, 96-122, DOI 10.1006/jdeq.2000.3814. MR1813101(2002b:76049)

[25] P. Joly and C. Tsogka, Numerical methods for treating unbounded media, Effective computational methods for wave propagation, Numer. Insights, vol. 5, Chapman \& Hall/CRC, Boca Raton, FL, 2008, pp. 425-472, DOI 10.1201/9781420010879.ch14. MR2404885 (2009f:65228)

[26] D. Leguillon and E. Sánchez-Palencia, Computation of Singular Solutions in Elliptic Problems and Elasticity, John Wiley \& Sons Ltd., Chichester, 1987. MR.995254 (90m:73015)

[27] M. Lenoir, M. Vullierme-Ledard, and C. Hazard, Variational formulations for the determination of resonant states in scattering problems, SIAM J. Math. Anal. 23 (1992), no. 3, 579-608, DOI 10.1137/0523030. MR1158823 (93c:35120)

[28] J.-J. Marigo and C. Pideri, The effective behavior of elastic bodies containing microcracks or microholes localized on a surface, International Journal of Damage Mechanics 20 (2011), no. 8, 1151-1177.

[29] D. Martin, Mélina, Bibliothèque de calculs éléments finis, http://anum-maths .univ-rennes1.fr/melina/danielmartin/melina/.

[30] V. Maz'ya, S. Nazarov, and B. Plamenevskij, Asymptotic theory of elliptic boundary value problems in singularly perturbed domains. Vol. I, Operator Theory: Advances and Applications, vol. 111, Birkhäuser Verlag, Basel, 2000. MR,1779977 (2001e:35044a)

[31] _ Asymptotic theory of elliptic boundary value problems in singularly perturbed domains. Vol. II, Operator Theory: Advances and Applications, vol. 112, Birkhäuser Verlag, Basel, 2000. MR:1779978 (2001e:35044b)

[32] V. G. Maz'ja and B. A. Plamenevskiŭ, Weighted spaces with inhomogeneous norms, and boundary value problems in domains with conical points (Russian), Elliptische Differentialgleichungen (Meeting, Rostock, 1977), Wilhelm-Pieck-Univ. Rostock, 1978, pp. 161-190. MR.540196 (81e:35045)

[33] G. Nakamura and G. Uhlmann, Global uniqueness for an inverse boundary problem arising in elasticity, Invent. Math. 118 (1994), no. 3, 457-474, DOI 10.1007/BF01231541. MR.1296354 (95i:35313)

[34] G. Nakamura and G. Uhlmann, Inverse problems at the boundary for an elastic medium, SIAM J. Math. Anal. 26 (1995), no. 2, 263-279, DOI 10.1137/S0036141093247494. MR.1320220 (96d:35146)

[35] D. Rabinovich, D. Givoli, J. Bielak, and T. Hagstrom, A finite element scheme with a high order absorbing boundary condition for elastodynamics, Comput. Methods Appl. Mech. Engrg. 200 (2011), no. 23-24, 2048-2066, DOI 10.1016/j.cma.2011.03.006. MR.2795161|(2012i:74043) 
[36] V. Bonnaillie-Noël; D. Brancherie; M. Dambrine; S. Tordeux and G. Vial, Effect of microdefects on structure failure. Coupling asymptotic analysis and strong discontinuity, Eur. J. Comput. Mech. 19 (2010), no. 1-3, 165-175 (English).

[37] D. Brancherie; M. Dambrine; G. Vial and P. Villon, Effect of surface defects on structure failure. A two-scale approach, Rev. Eur. Méc. Numér. 17 (2008), no. 5-7, 613-624 (English).

[38] M. S. Vogelius and D. Volkov, Asymptotic formulas for perturbations in the electromagnetic fields due to the presence of inhomogeneities of small diameter, M2AN Math. Model. Numer. Anal. 34 (2000), no. 4, 723-748, DOI 10.1051/m2an:2000101. MR.1784483 (2001f:78024)

[39] O. C. Zienkiewicz and P. Bettess, Infinite elements in the study of fluid-structure interaction problems, Computing methods in applied sciences (Second Internat. Sympos., Versailles, 1975), Springer, Berlin, 1976, pp. 133-172. Lecture Notes in Phys., Vol. 58. MR0443580 (56 \#1949)

IRMAR - UMR6625, Ens Rennes, Univ. Rennes 1, CNRS, UEB, AV. Robert Schuman, 35170 BRUZ, FRANCE

E-mail address: bonnaillie@math.cnrs.fr

LMAP - UMr5142, Université de Pau et des Pays de l'Adour, AV. de l'Université, BP 1155, 64013 Pau Cedex, France

E-mail address: marc.dambrine@univ-pau.fr

LMJL - UMr6629, Université de NAntes, 2 Rue De LA Houssinière, BP 92208, 44322 Nantes Cedex 3, France

E-mail address: frederic.herau@univ-nantes.fr

Université de Lyon, CNRS UMR 5208, École Centrale de Lyon, Institut Camille Jordan, 36 avenue Guy de Collongue, 69134 Écully cedex, France

E-mail address: gregory.vial@ec-lyon.fr 